\title{
Increased flux through the mevalonate pathway mediates fibrotic repair without injury
}

\author{
Jennifer L. Larson-Casey, ${ }^{1}$ Mudit Vaid, ${ }^{1}$ Linlin Gu, ${ }^{1}$ Chao He, ${ }^{1}$ Guo-Qiang Cai, ${ }^{1}$ Qiang Ding, ${ }^{1}$ Dana Davis, ${ }^{1}$ Taylor F. Berryhill, ${ }^{2}$ \\ Landon S. Wilson, ${ }^{2}$ Stephen Barnes, ${ }^{2}$ Jeffrey D. Neighbors, ${ }^{3,4,5}$ Raymond J. Hohl, ${ }^{3,4,5}$ Kurt A. Zimmerman, ${ }^{6}$ Bradley K. Yoder, ${ }^{6}$ \\ Ana Leda F. Longhini, ${ }^{7}$ Vidya Sagar Hanumanthu, ${ }^{7}$ Ranu Surolia, ${ }^{1}$ Veena B. Antony, ${ }^{1}$ and A. Brent Carter ${ }^{1,8}$ \\ 'Department of Medicine, Division of Pulmonary, Allergy, and Critical Care Medicine, and ${ }^{2}$ Targeted Metabolomics and Proteomics Laboratory, University of Alabama at Birmingham, Birmingham, Alabama, \\ USA. ${ }^{3}$ Department of Medicine, and ${ }^{4}$ Department of Pharmacology, College of Medicine, Penn State University, Hershey, Pennsylvania, USA. ${ }^{5}$ Penn State Cancer Institute, Hershey, Pennsylvania, USA. \\ ${ }^{6}$ Department of Cell, Developmental, and Integrative Biology, and 'Division of Clinical Immunology and Rheumatology, University of Alabama at Birmingham, Birmingham, Alabama, USA. ${ }^{8}$ Birmingham \\ Veterans Administration Medical Center, Birmingham, Alabama, USA.
}

\begin{abstract}
Macrophages are important in mounting an innate immune response to injury as well as in repair of injury. Gene expression of Rho proteins is known to be increased in fibrotic models; however, the role of these proteins in idiopathic pulmonary fibrosis (IPF) is not known. Here, we show that BAL cells from patients with IPF have a profibrotic phenotype secondary to increased activation of the small GTPase Rac1. Rac1 activation requires a posttranslational modification, geranylgeranylation, of the C-terminal cysteine residue. We found that by supplying more substrate for geranylgeranylation, Rac1 activation was substantially increased, resulting in profibrotic polarization by increasing flux through the mevalonate pathway. The increased flux was secondary to greater levels of acetyl-CoA from metabolic reprogramming to $\beta$ oxidation. The polarization mediated fibrotic repair in the absence of injury by enhancing macrophage/fibroblast signaling. These observations suggest that targeting the mevalonate pathway may abrogate the role of macrophages in dysregulated fibrotic repair.
\end{abstract}

\section{Introduction}

Pulmonary fibrosis is a progressive disease characterized by irreversible scarring of the lungs. The disease is associated with significant morbidity and mortality, and the median life expectancy is 3-5 years after diagnosis for certain forms of pulmonary fibrosis, such as idiopathic pulmonary fibrosis (IPF) (1). Although the recently approved antifibrotic therapies for IPF reduce the rate of lung function decline, neither drug is associated with improvements in quality of life or survival $(2,3)$.

A chronic inflammatory process of the lungs was previously considered the underlying mechanism driving IPF (4), but the inflammatory process is no longer considered an important component of IPF pathology or a factor contributing to the pathogenesis of the disease. Accumulating evidence suggests that IPF is a fibrotic process resulting from recurrent injury to the alveolar epithelium followed by dysregulated repair processes leading to fibrosis (5-7).

Macrophages are important not only in mounting an innate immune response to foreign agents but also in the repair of injury. In fibrotic disorders of the lung, monocyte-derived macrophages (MDMs) have a decisive role in fibrotic repair of the injured lung. Macrophages play an integral role in the normal resolution of organ injury but can also contribute to fibrotic repair depending on the phenotype of the macrophages in the lungs. These diver-

Conflict of interest: The authors have declared that no conflict of interest exists. Copyright: (5) 2019, American Society for Clinical Investigation.

Submitted: February 6, 2019; Accepted: August 13, 2019; Published: October 14, 2019. Reference information: J Clin Invest. 2019;129(11):4962-4978.

https://doi.org/10.1172/JCl127959. gent functions are dependent on their ability to switch between phenotypically distinct subpopulations. Profibrotic macrophages express characteristic marker proteins associated with profibrotic changes, such as (a) production of antiinflammatory and profibrotic immune effectors, (b) upregulation of L-arginine metabolism by arginase 1 to generate polyamines and proline, and (c) alteration of extracellular matrix dynamics. Macrophages have mixed phenotypes in complex pathological conditions, such as lung injury and fibrosis, and polarize to a predominant phenotype depending on the duration and stage of injury repair (8-11).

Several pathways are known to regulate macrophage polarization $(9,12-14)$; however, there is no link between macrophage polarization and the mevalonate pathway, which regulates sterol biosynthesis. Several pharmaceutical agents have been designed to target enzymes in the mevalonate biosynthetic pathway as therapy for multiple conditions, including hypercholesterolemia, cardiovascular disease, cancer, osteoporosis, neurodegenerative disorders, and several infectious diseases (15-17). Isoprenoid derivatives of the nonsterol portion of the mevalonate pathway are required for the posttranslational modification of several proteins, including the Rho GTPases, leading to the regulation of various cellular functions (18). Although Rho GTPase proteins are known to be increased in fibrotic models (19), the role of these proteins in IPF is poorly understood. In the present study, we found that increased flux through the mevalonate pathway enhanced the posttranslational modification of Rac1 that promotes macrophage/fibroblast signaling. Moreover, augmented Rac1 activity in MDMs mediated lung fibrosis in the absence of lung injury and exacerbated fibrosis in the presence of injury. 
A

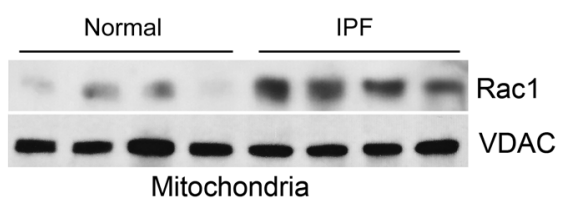

D
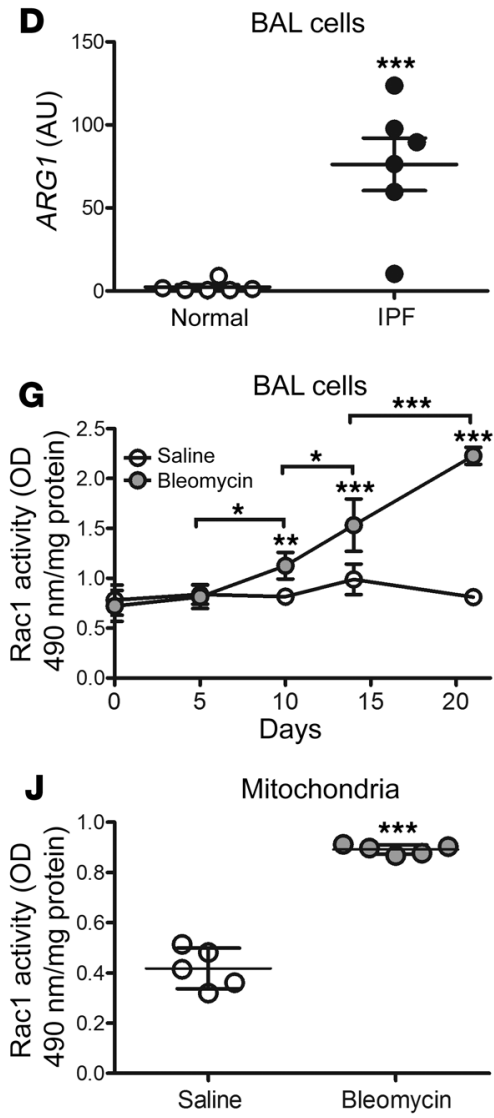

B

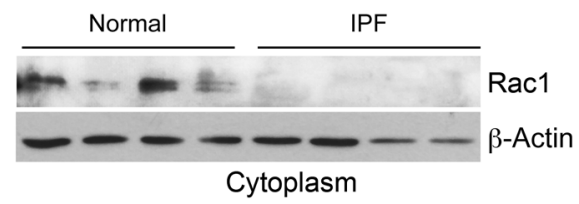

C

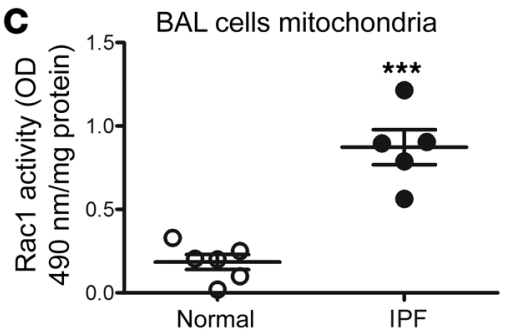

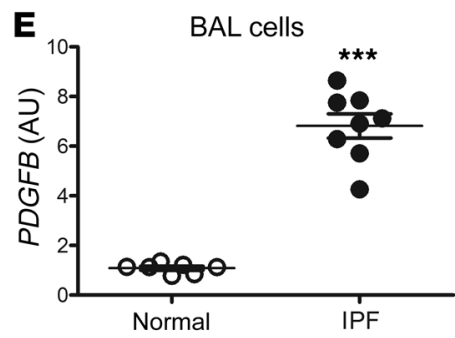

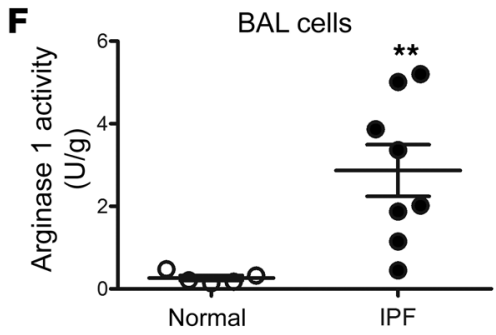

H

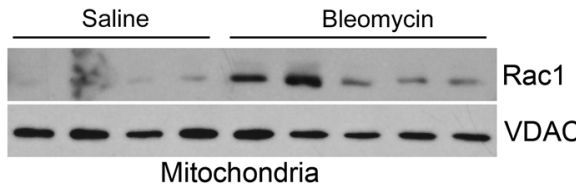

I

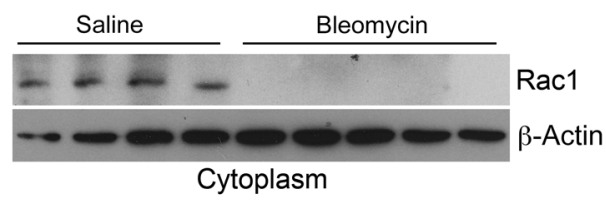

BALF

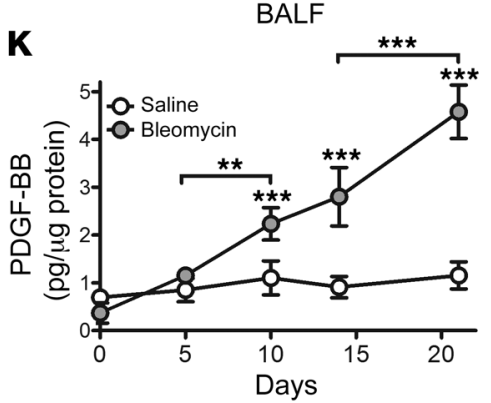

Figure 1. Mitochondrial Rac1 activity is increased in IPF BAL cells. Immunoblot analysis of Rac1 expression in isolated (A) mitochondria or (B) cytosol fractions from BAL cells obtained from healthy subjects $(n=4)$ or patients with IPF $(n=4)$. (C) Mitochondrial Rac1 activity in BAL cells from healthy subjects $(n=6)$ or patients with IPF $(n=5)$. BAL cells from healthy subjects $(n=6-7)$ or patients with IPF $(n=6-8)$ were analyzed for mRNA expression of (D) ARG1 and (E) PDGFB. (F) Arginase 1 activity in BAL cells from healthy subjects $(n=5)$ or patients with IPF $(n=8)$. (C) Rac1 activity in BAL cells isolated at the indicated time points from mice exposed to saline or bleomycin ( $n=3-4$ mice/time point). Immunoblot analysis of Rac1 expression in isolated (H) mitochondria or (I) cytosolic fractions from BAL cells obtained from mice treated with saline $(n=4)$ or bleomycin $(n=5)$. (J) Mitochondrial Rac1 activity in BAL cells from saline- $(n=5)$ or bleomycin-exposed $(n=5)$ mice. (K) PDGF-BB and (L) IL-10 were measured in BALF from saline- or bleomycin-exposed mice at the indicated time points ( $n=3-4$ mice/time point). Values indicate the mean \pm SEM. ${ }^{*} P<0.05,{ }^{* *} P<0.001$, and ${ }^{* * *} P<0.0001$, by 2 -tailed Student's $t$ test (C-F and $\mathbf{J}$ ) and 2-way ANOVA with Bonferroni's post test (C, $\mathbf{K}$, and $\mathbf{L}$ ).

\section{Results}

Mitochondrial Rac1 activity is increased in IPF bronchoalveolar lavage cells. To determine the role of the mevalonate pathway in patients with IPF, we asked whether Rac1, a Rho GTPase implicated in fibrotic disorders (20-22), is present in the mitochondria of IPF bronchoalveolar (BAL) cells. We observed that Rac1 localization in mitochondria was increased in BAL cells from patients with IPF (Figure 1A), whereas healthy subjects retained Rac1 in the cytosol (Figure 1B). The posttranslational modification - geranylgeranylation - of Rac1 is required for its activation and localization to the mitochondrial intermembrane space (22). Mitochondria isolated from IPF BAL cells showed a nearly 5 -fold increase in Rac1 activity compared with cells from healthy volunteers (Figure 1C).

Because macrophages in chronic disease exhibit apoptosis resistance, which is associated with disease progression due to polarization to a profibrotic phenotype (9), we found that IPF BAL cells had significantly increased mRNA expression of ARG1 (Figure 1D) and PDGFB (Figure 1E). Additionally, IPF BAL cells showed a 10-fold increase in arginase 1 activity compared with cells from healthy volunteers (Figure 1F). In contrast, TNF and NOS2 expression levels were dramatically reduced in BAL cells from patients with IPF (Supplemental Figure 1, A and B; supple- 
mental material available online with this article; https://doi. org/10.1172/JCI127959DS1).

To determine whether lung injury promotes mitochondrial Rac1 localization, we harvested BAL cells from WT mice after bleomycin-induced injury, and monocytic cells were the primary cell in the BAL at all time points (Supplemental Figure 1C). We found that Rac1 activity was significantly increased in bleomycin-injured BAL cells isolated 10 days after exposure and that activity progressively increased in a time-dependent manner (Figure 1G). Rac1 expression was increased in isolated mitochondria from bleomycininjured mice, whereas Rac1 was localized in the cytosol in the saline controls 21 days after exposure (Figure $1, \mathrm{H}$ and I). BAL cells with mitochondrial Rac1 localization showed a 2-fold increase in mitochondrial Rac1 activity (Figure 1J). These cells polarized to a profibrotic phenotype with significantly greater PDGF-BB and IL-10 expression that increased in a time-dependent manner (Figure 1, $\mathrm{K}$ and L); however, the amounts of TNF- $\alpha$ in BAL fluid (BALF) and Nos 2 gene expression were significantly lower in cells from bleomycin-injured mice (Supplemental Figure 1, D and E).

Increasing Rac1 activity by augmenting isoprenylation promotes lung fibrosis. Because of the significant difference in mitochondrial Rac1 activity in subjects with IPF and bleomycin-injured mice, we questioned whether this difference was critical in the pathogenesis of pulmonary fibrosis. To increase mitochondrial Rac1 activity, THP-1 cells were treated with geranylgeraniol (GGOH), an analog of geranylgeranyl diphosphate (GGDP), to increase Rac1 geranylgeranylation and, thus, activation (Figure $2 \mathrm{~A}$ ). We found that GGOH increased Rac1 mitochondrial localization, which was further enhanced by overexpression of $\mathrm{Rac}_{\mathrm{WT}}$ (Figure 2, B and C). Cytosolic Rac1 content was reduced in cells treated with GGOH (Supplemental Figure 2A). Providing more substrate for Rac1 isoprenylation with GGOH increased mitochondrial Rac1 activity to a level similar to that seen in macrophages expressing Rac $1_{\mathrm{WT}}$, and GGOH led to a significantly greater increase in activity in $\mathrm{Rac}_{\mathrm{WT}}$-expressing macrophages (Figure 2D). To confirm that this was secondary to geranylgeranylation of Rac1, lysates were separated into aqueous (unprenylated) and detergent (isoprenylated) phases to determine Rac1 isoprenylation status. Rac1 was retained in the detergent phase when exposed to GGOH (Figure 2E). The increase in isoprenylation was specific for Rac1, as Rac2, the other prominent GTPase in macrophages, remained in the aqueous phase in cells treated with GGOH (Supplemental Figure 2B).

To further validate the importance of the posttranslational modification, we evaluated Rac1 activity by mutation of the C-terminal cysteine residue ( $\left.\mathrm{Cys}^{189}\right)$, the site of geranylgeranylation. Mitochondrial Rac1 activity in THP-1 cells expressing Rac1 ${ }_{\mathrm{C} 189 \mathrm{~S}}$ was markedly lower than that seen in the empty control (Supplemental Figure 2C). Similarly, silencing GGDP synthase (GGDPS), which enzymatically generates GGDP, abolished Rac1 activity in THP-1 cells expressing Rac1 $1_{\mathrm{WT}}$ (Supplemental Figure 2D). Increasing Rac1 activity with GGOH led to a significant increase in $T g f b 1$, Retnla, and Pdgfb gene expression (Supplemental Figure 2, E-G).

To investigate the pathological significance of enhanced mitochondrial Rac1 activation in macrophages, mice were exposed to saline or bleomycin. Once fibrotic repair was initiated 10 days after exposure, mice received vehicle or GGOH via subcutaneously implanted miniosmotic pumps. GGOH increased the number of cells in the BALF to a level similar to that observed with bleomycin, and the combination of bleomycin and GGOH treatment led to a further increase (Supplemental Figure 2H). Monocytic cells were the primary cell type in the BALF, regardless of exposure (Supplemental Figure 2I).

GGOH treatment led to an increase in mitochondrial localization of Rac1 that was further increased in bleomycin-injured mice receiving GGOH (Figure $2 \mathrm{~F}$ ). The opposite trend was observed in the cytoplasmic fractions (Supplemental Figure 2J). The increase in mitochondrial Rac1 localization resulted in significantly enhanced Rac1 isoprenylation (Figure 2G) and mitochondrial Rac1 activity in BAL cells from GGOH-treated mice (Figure 2H). Rac1 activity was further increased by greater than 4 -fold in bleomycin-injured mice receiving GGOH treatment. The increase in mitochondrial Rac1 activity was associated with an increase in Tgfb1, Pdgfb, and Retnla mRNA expression in BAL cells from GGOH-treated and bleomycin-injured mice (Figure 2I and Supplemental Figure 2, K and L). The phenotypic switching of macrophages to a profibrotic phenotype led to an increased concentration of active TGF- $\beta 1$ and Ym- 1 in BALF from GGOH-treated and bleomycin-injured mice (Figure 2, J and K).

To determine whether the enhanced Rac1 activity by GGOH altered the pathogenesis of fibrotic repair, histological analysis showed that lungs from mice treated with GGOH alone had an increase in collagen deposition that was similar to that seen with bleomycin-induced injury (Figure 2L). The bleomycin-injured mice receiving GGOH showed even greater architectural destruction and increased collagen deposition. These observations were confirmed biochemically (Figure 2M); however, administration GGOH did not alter Rac1 activity, Tgfb1 or Col1a1 gene expression, or collagen content in other organs, including the liver and kidneys (Supplemental Figure 2, M-Q).

To extend these observations to other experimental models of fibrosis, we found that chrysotile asbestos-exposed mice showed increased mitochondrial Rac1 expression that was further increased in GGOH-treated mice (Supplemental Figure 2R). Rac1 remained in the cytoplasm in mice receiving vehicle (Supplemental Figure 2S). Chrysotile exposure led to increased collagen deposition similar to what was observed in the lungs from mice treated with GGOH alone, and the combination showed further architecture destruction and increased collagen deposition (Supplemental Figure 2, $\mathrm{T}$ and $\mathrm{U}$ ). Taken together, increasing geranylgeranylation and mitochondrial localization of Rac1 with GGOH significantly amplified Rac1 activity, polarized macrophages to a profibrotic phenotype, and exacerbated fibrotic repair.

GGOH-mediated lung fibrosis occurs in the absence of injury. Because the current paradigm supports the notion that alveolar epithelial cell (AEC) injury and apoptosis initiate pulmonary fibrosis development $(5,23)$, we asked whether AECs were injured by GGOH. We observed reduced ER stress (Supplemental Figure 3A) and significantly less caspase-3 activity in AECs from mice treated with GGOH compared with AECs from vehicle-treated mice (Figure 3A). Bleomycin-injured mice showed significantly increased caspase- 3 activity, whereas AECs from bleomycin-exposed mice that received GGOH had a marked reduction in caspase-3 activity. In lung tissue, AECs were present in GGOH-treated mice and were not $\mathrm{TUNEL}^{+}$(Figure 3B). Although bleomycin-exposed lungs 
A

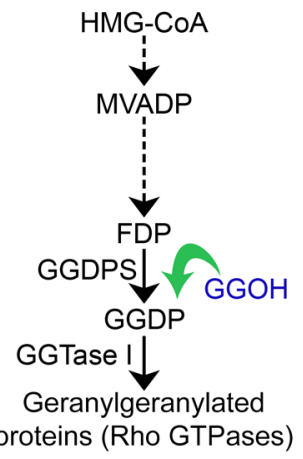

B

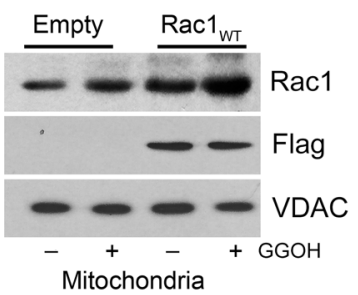

C

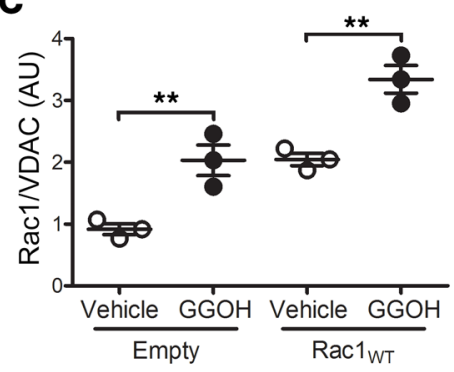

D

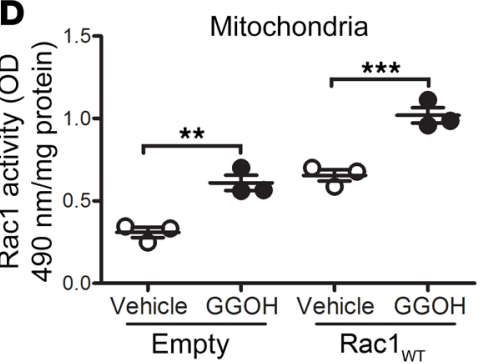

E

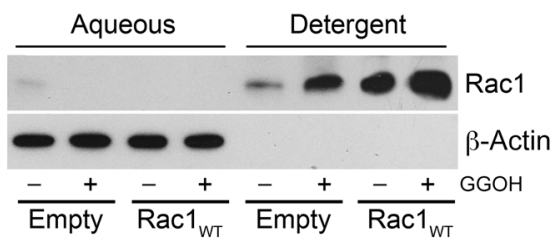

F

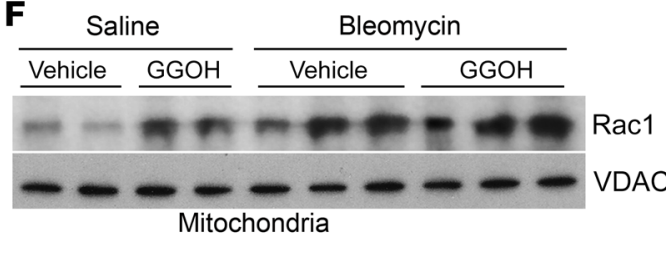

G

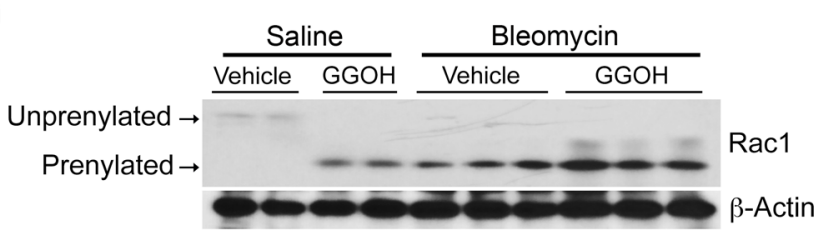

H

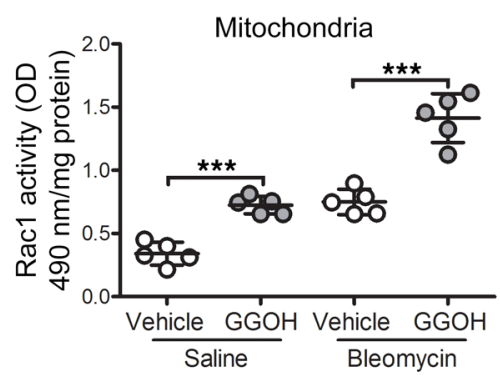

I

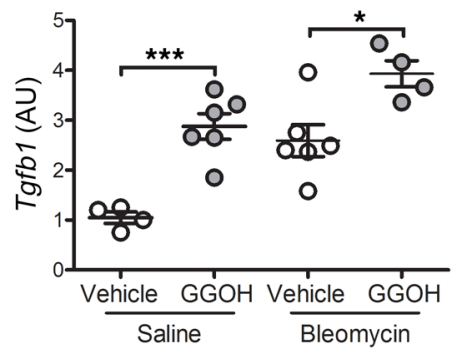

J

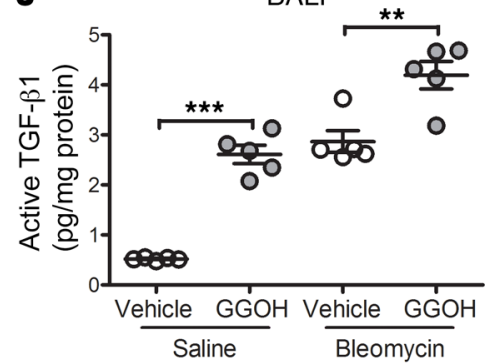

K

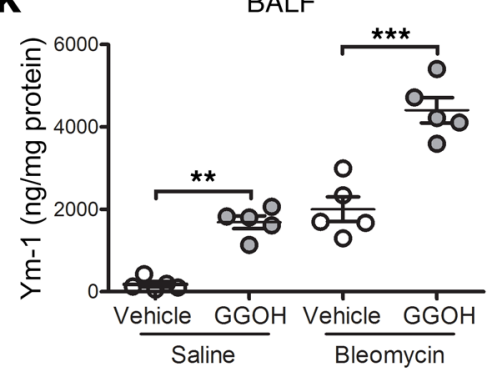

$\mathbf{L}$

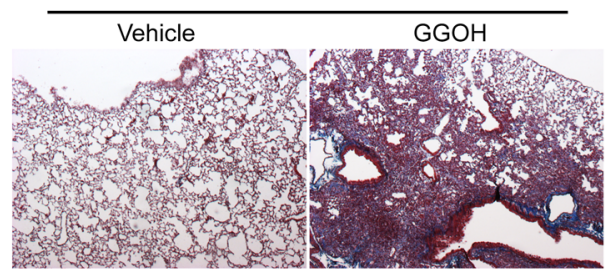

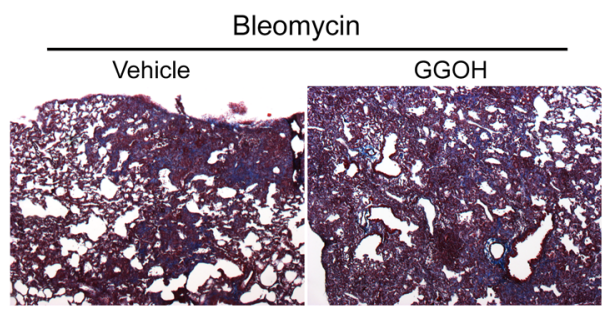

M

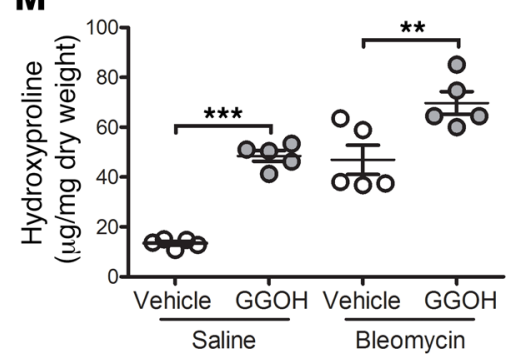

Figure 2. Increasing Rac1 activity by augmentation of isoprenylation promotes lung fibrosis. (A) Schematic diagram of the mevalonate pathway. HMG-CoA, 3-hydroxy-3-methylglutaryl coenzyme A; MVADP, mevalonate 5-diphosphate. (B) Immunoblot analysis and (C) quantification of isolated mitochondria from THP-1 cells expressing empty control or Rac $1_{W T}$ and treated with vehicle or GGOH $(50 \mu M)(n=3)$. (D) Mitochondrial Rac1 activity in transfected MH-S cells treated with vehicle or $\mathrm{GGOH}(n=3)$. (E) Immunoblot analysis of transfected macrophages expressing empty control or Rac1 ${ }_{W T}$ and treated with vehicle or GGOH. Cells were separated into aqueous (unprenylated) or detergent (prenylated) fractions. Ten days after exposure of WT mice to saline or bleomycin, pumps containing vehicle or GGOH were implanted s.c., and the mice were sacrificed 11 days later. (F) Mitochondrial Rac1 immunoblot analysis of isolated MDMs. (C) Isoprenylation status of Rac1 in isolated MDMs. (H) Mitochondrial Rac1 activity ( $n=5$ /group). (I) Tgfb1 mRNA expression (saline, vehicle $n=4$; saline, GGOH $n=6$; bleomycin, vehicle $n=6$; bleomycin, GGOH $n=4$ ). (J) Active TCF- $\beta 1$ and (K) Ym-1 expression in BALF ( $n=5 /$ group). (L) Representative lung histology images with Masson's trichrome staining ( $n=5$ /group). Original magnification, $\times 2.5$. (M) Hydroxyproline content $\left(n=5\right.$ /group). Values indicate the mean \pm SEM. ${ }^{*} P<0.05$, ${ }^{*} P<0.001$, and ${ }^{* * *} P<0.0001$, by 1-way ANOVA followed by Tukey's multiple comparisons test. 
A

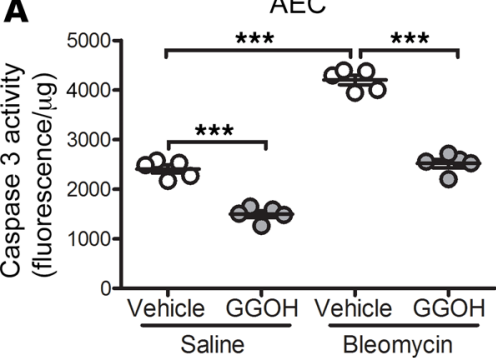

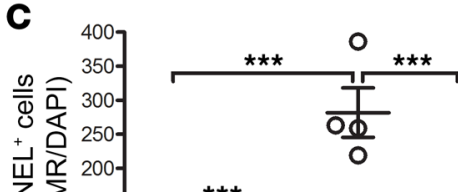

\.

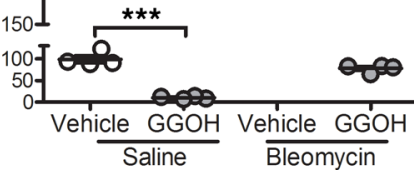

D

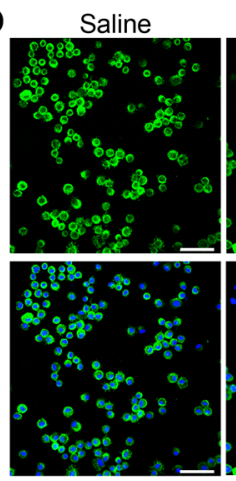

Bleomycin

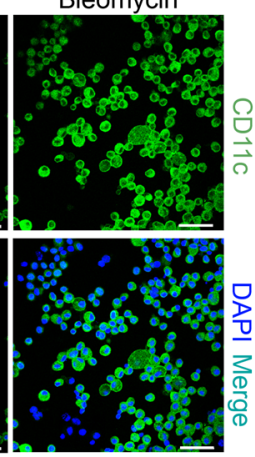

H

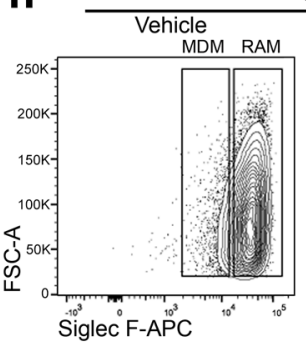

J

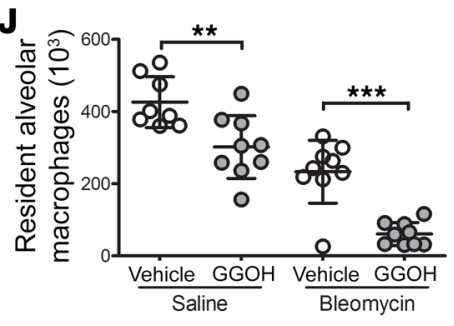

M

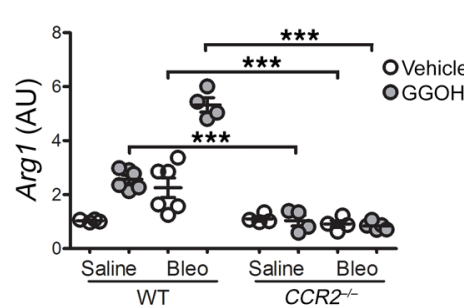

B

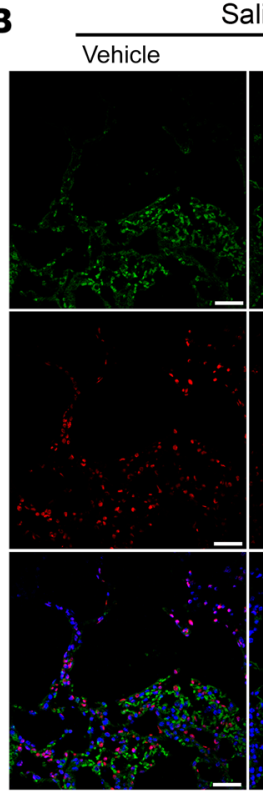

Saline
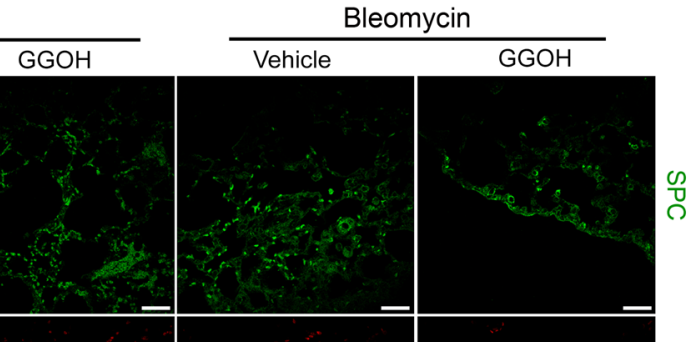

E

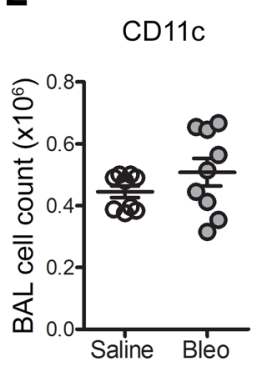

F Saline
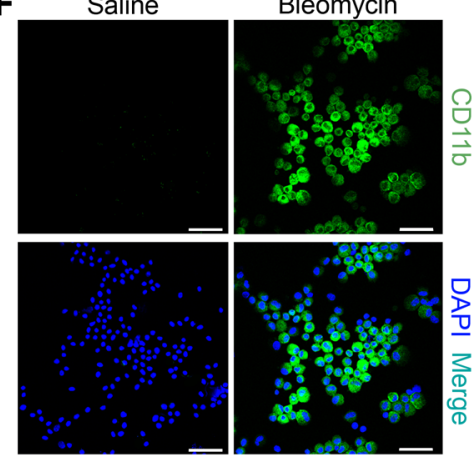

G

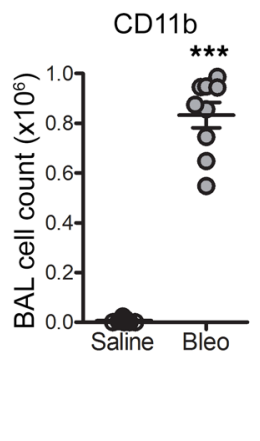

Saline

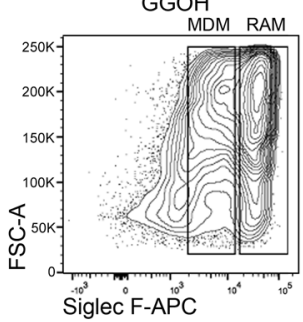

$\mathbf{K}$
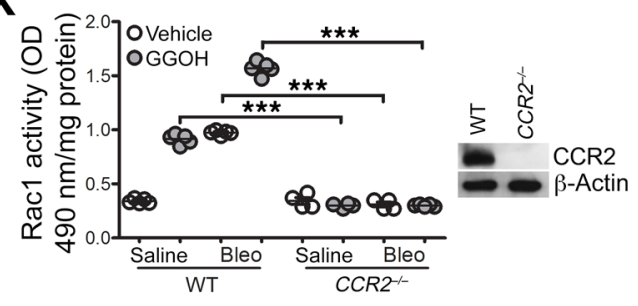
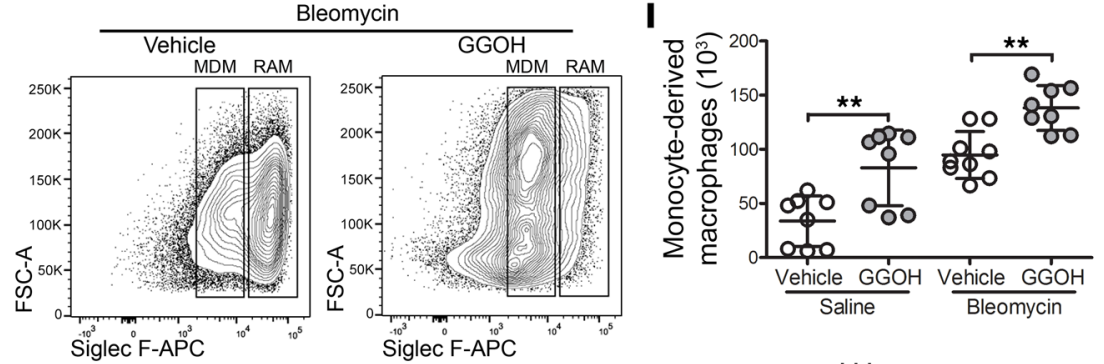
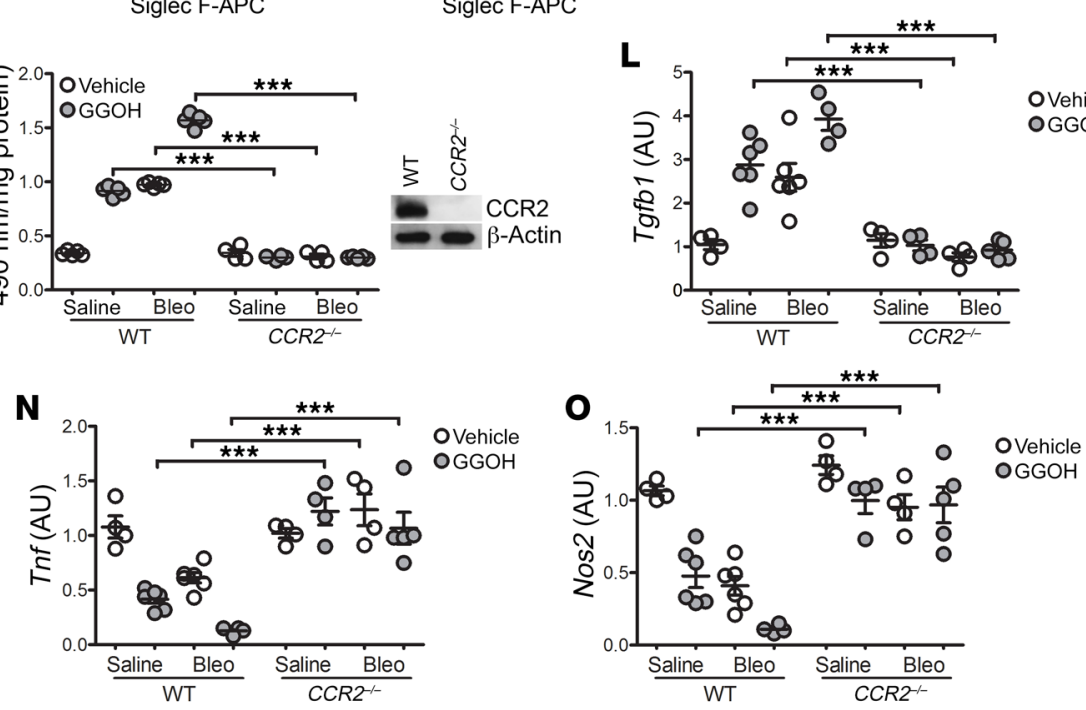
Figure 3. GGOH-mediated lung fibrosis occurs in the absence of injury. Ten days after exposure of WT mice to saline or bleomycin, pumps containing vehicle or $\mathrm{GGOH}$ were s.c. implanted; mice were sacrificed 11 days later. (A) Caspase-3 activity in AECs ( $n=5$ /group). (B) Representative images of lung IHC for SPC (green), TUNEL (red), and DAPI (blue) ( $n=4$ per group) and (C) quantification of TUNEL ${ }^{+}$cells from B ( $n=4 /$ group). Scale bars: $20 \mu \mathrm{m}$. WT mice were exposed to saline or bleomycin. Confocal imaging and total number of BAL cells stained with ( $\mathbf{D}$ and $\mathbf{E})$ CD11c $(n=$ 5-9) and (F and G) CD11b ( $n=5-9)$, together with DAPI. Scale bars: $50 \mu \mathrm{m}$ Ten days after exposure of WT mice to saline or bleomycin (Bleo), pumps containing vehicle or $\mathrm{GGOH}$ were s.c. implanted, and lungs were extracted 11 days later. (H) Representative flow cytometric plots of MDMs (CD45 ${ }^{+}$

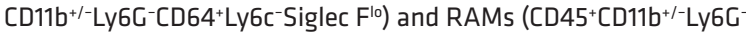
CD64+Ly6c ${ }^{-}$Siglec $F^{\text {hi)}}$ ). FSC-A, forward scatter area. Total numbers of (I) MDMs and (J) RAMs from BAL. WT or CCR2 $2^{-1-}$ mice were exposed and treated as described above. (K) Rac1 activity $(n=4-5)$ and mRNA expression of (L) Tgfb1 ( $n=4-5)$, (M) $\operatorname{Arg} 1(n=4-5)$, (N) $\operatorname{Tnf}(n=4-5)$, and (0) Nos2 $(n=4-5)$. Inset in K shows CCR2 immunoblot analysis. Values indicate the mean \pm SEM. ${ }^{* *} P<0.001$ and ${ }^{* * *} P<0.0001$, by 2 -tailed Student's $t$ test (E and $\mathbf{G}$ ) and 1-way ANOVA followed by Tukey's multiple comparisons test (A, C, and I-0)

show increased TUNEL ${ }^{+} \mathrm{AECs}$, GGOH significantly reduced the number of TUNEL ${ }^{+}$cells in bleomycin-injured mice (Figure 3C). AEC apoptosis resistance was not dependent on RhoA or Rac1 activity (Supplemental Figure 3, B and C). These results indicate that increased Rac1 activity in macrophages promotes fibrotic repair in the absence of AEC injury.

Recent evidence suggests that MDMs are responsible for pulmonary fibrosis (24). We determined that there were similar numbers of $\mathrm{CD} 11 \mathrm{c}^{+}$cells from saline-treated and bleomycininjured mice (Figure 3, D and E); however, we observed a significant increase in CD11b ${ }^{+}$cells (Figure 3, F and G) in BAL cells from mice that received bleomycin. We questioned the source of the BAL cells from GGOH-treated mice. Bleomycin-induced injury significantly increased the number of MDMs compared with saline-treated mice, and recruitment was further enhanced in bleomycin-injured mice that received GGOH (Figure 3, $\mathrm{H}$ and I). Mice receiving bleomycin showed a significant reduction in the percentage of resident alveolar macrophages (RAMs), and the combination of bleomycin and GGOH reduced the levels further (Figure 3, $\mathrm{H}$ and J, and Supplemental Figure 3D). Administration of GGOH increased Rac1 activity in isolated lung monocytic cells, whereas monocytic cells isolated from the kidney and liver showed no alterations in Rac1 or Rac2 activity (Supplemental Figure 3, E and F).

The increase in MDMs in GGOH-treated and bleomycininjured mice correlated with greater MCP-1 levels in BALF, suggesting increased recruitment (Supplemental Figure 3G). Depletion of circulating monocytes using $C C R 2^{-/}$mice resulted in a significant reduction in Rac1 activity that was similar to that seen with WT saline controls (Figure 3K). The absence of MDMs inhibited profibrotic polarization and promoted a proinflammatory phenotype (Figure 3, L-O). Although CCR2- mice were protected from bleomycin- or GGOH-mediated fibrosis (Supplemental Figure 3H), AECs from bleomycin-injured $C C R 2^{--}$mice showed increased caspase- 3 activity that was not altered in mice receiving GGOH; however, enhancing Rac1 activation with GGOH significantly decreased caspase-3 activity in AECs from bleomycin-exposed WT mice (Supplemental Figure 3I). These data suggest that an increase in geranylgeranylation and activation of Rac1 occur in lung MDMs and thereby contribute to a fibrotic phenotype. These data also suggest that targeting the posttranslational modification of Rac1 in MDMs may have a curative effect on fibrosis progression.

Mitochondrial ROS is required for Rac1-mediated profibrotic polarization of BAL cells. Because mitochondrial localization of Rac1 is required for mitochondrial ROS (mtROS) generation (22, 25), we found that BAL cells from patients with IPF had a 4-fold increase in mtROS generation (Figure 4A). Similarly, MDMs isolated from bleomycin-injured mice showed increased mtROS generation (Figure 4B). Given that GGOH enhanced mitochondrial Rac1 expression and activity, GGOH induced mtROS, which was further enhanced in GGOH-treated THP-1 cells expressing Rac1 $_{\mathrm{WT}}$ (Figure $4 \mathrm{C}$ ). The generation of mtROS was increased in MDMs from mice receiving GGOH compared with those from vehicle-treated mice, and mtROS was further enhanced in bleomycin-injured mice treated with GGOH (Figure 4D). Increased mtROS was Rac1 dependent, as BAL cells isolated from mice harboring a conditional deletion of Rac1 in macrophages ( $\mathrm{Racl}^{-1-}$ Lyz2-Cre) showed a significant reduction in mtROS, and bleomycin exposure had no effect (Figure 4E).

Generation of mtROS is critical for the polarization of macrophages to a profibrotic phenotype, and the transcription factor STAT6 regulates this process $(8,26)$. Phosphorylation of nuclear STAT6 (p-STAT6) was increased in THP-1 cells treated with GGOH or expressing $\mathrm{Rac1}_{\mathrm{WT}}$, and the combination further enhanced p-STAT6 content in the nucleus (Figure $4 \mathrm{~F}$ and Supplemental Figure 4A). Because HIF-2 $\alpha$ promotes profibrotic polarization (27) and STAT6 is redox regulated (8) and modifies PPAR $\gamma$ and Krüppel-like factor 4 (KLF4) expression $(28,29)$, we asked whether Rac1-mediated mtROS modulated these transcription factors. We found that $\mathrm{Rac}_{\mathrm{WT}}$ regulated the nuclear expression of these transcription factors and that GGOH increased PPAR $\gamma$, KLF4, and HIF- $2 \alpha$ nuclear localization to a greater extent. To demonstrate the association of mtROS with Rac1-mediated regulation of these transcription factors, inhibition of mtROS by silencing the mitochondrial complex III iron-sulfur protein Rieske (Figure 4G) abolished nuclear localization of STAT6, PPAR $\gamma, \mathrm{KLF} 4$, and HIF-2 $\alpha$ in THP-1 cells expressing Rac1 ${ }_{\mathrm{WT}}$ (Figure $4 \mathrm{H}$ ). Moreover, THP-1 cells expressing dominant-negative Rac1 inhibited the nuclear localization of KLF4 and HIF- $2 \alpha$ (Figure 4I). The inability of THP- 1 cells expressing Rac1 C189S $_{\text {to generate mtROS (Supplemental Figure 4B) }}$ led to increased STAT1 activation and nuclear localization of the p-p65 subunit of NF- $\mathrm{kB}$ and HIF-1 $\alpha$ (Supplemental Figure 4C), actions that polarize macrophages to a proinflammatory phenotype. Furthermore, these results were confirmed by inhibiting mtROS with MitoTEMPO (Supplemental Figure 4D).

The nuclear localization of STAT6, KLF4, PPAR $\gamma$, and HIF- $2 \alpha$ promotes the utilization of L-arginine by arginase $1(27,29,30)$. Silencing KLF 4 or HIF- $2 \alpha$ significantly inhibited arginase 1 promoter activity below control levels in the presence of Rac1 ${ }_{\mathrm{WT}}$ (Figure 4J), suggesting that KLF 4 and HIF-2 $\alpha$ are critical downstream targets of Rac1. Rac1 isoprenylation with GGOH treatment or Rac1 $1_{\mathrm{WT}}$ expression increased Arg1 promoter activity, whereas MH-S cells expressing Rac1 $_{\text {C189S }}$ resulted in significantly reduced promoter activity, regardless of treatment (Figure 4K). Similar results were obtained with Retnla-driven luciferase activity (Supplemental Figure 4E). 

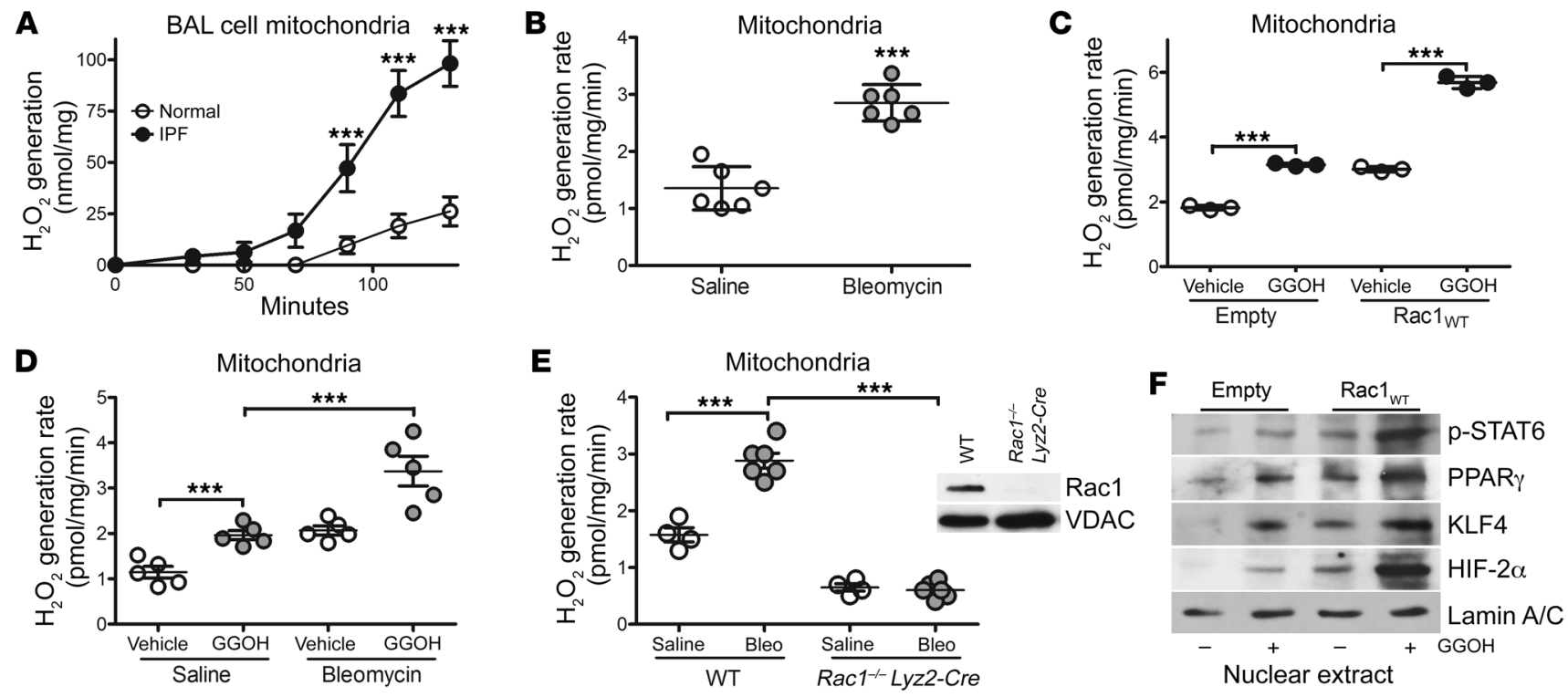

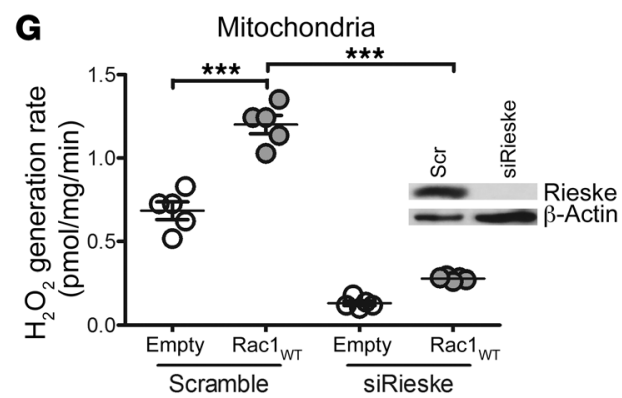

H

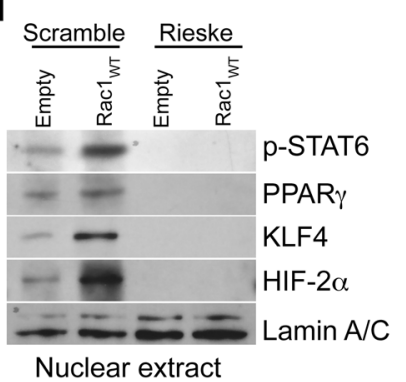

K

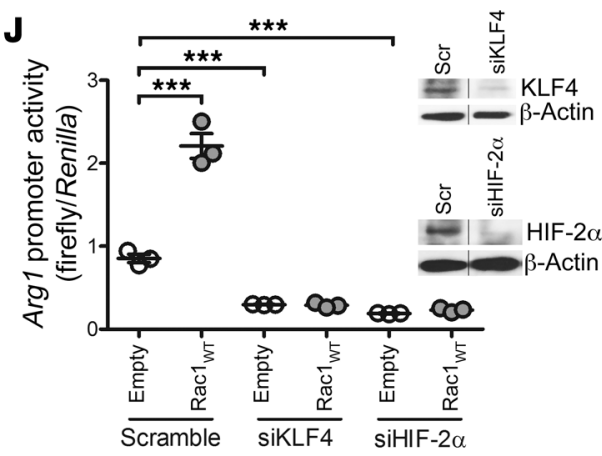

I

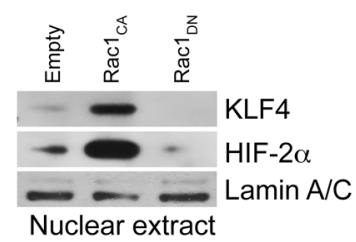

L

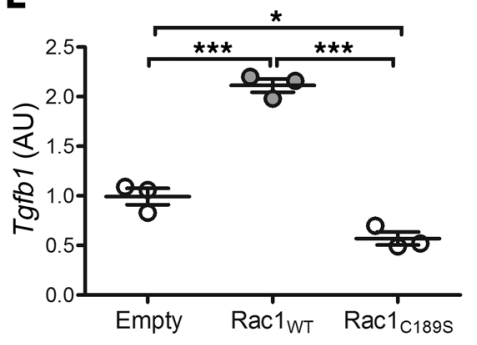

M

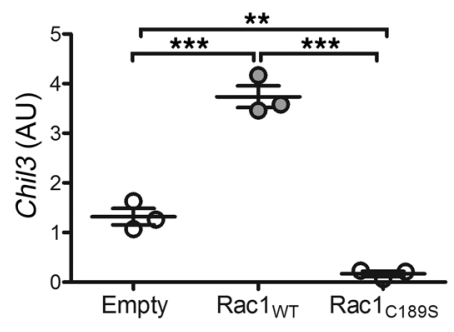

$\mathbf{N}$

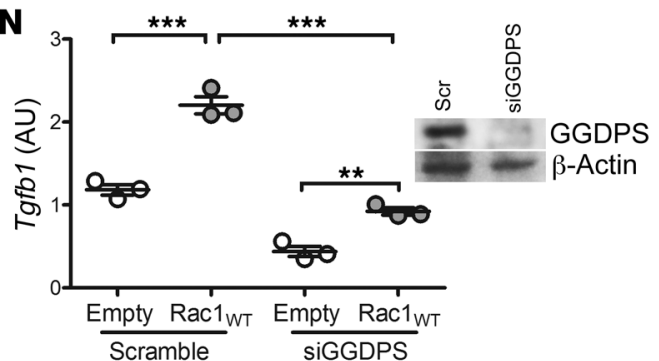

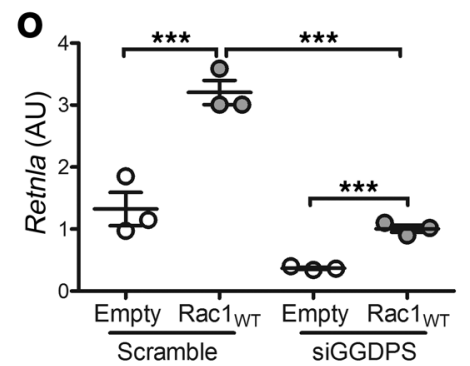


Figure 4. $m$ tROS is required for Rac1-mediated profibrotic polarization of BAL cells. mtROS generation in BAL cells from $(\mathbf{A})$ healthy subjects $(n=6)$ or patients with IPF $(n=6)$ and from (B) saline- $(n=6)$ or bleomycinexposed $(n=6)$ mice. (C) mtROS generation in THP-1 cells expressing empty control or Rac1 ${ }_{W T}$ and treated with vehicle or GGOH $(n=3)$. (D) Ten days after exposure of mice to saline or bleomycin, pumps containing vehicle or GGOH were implanted s.c. into mice, and the mice were sacrificed 11 days later. Data show mtROS generation in isolated MDMs ( $n=5$ mice/ group). (E) mtROS generation in isolated BAL cells from bleomycin- or saline-exposed WT or Rac1 ${ }^{-/-}$Lyz2-Cre mice (saline, $n=4$ mice; bleomycin $n=6$ mice). Inset shows immunoblot analysis. (F) Nuclear immunoblot analysis of transfected THP-1 cells treated with vehicle or $\mathrm{GGOH}(n=3)$. (C) mtROS generation in transfected THP-1 cells $(n=5)$. Inset shows Rieske immunoblot analysis. Nuclear immunoblot analysis of THP-1 cells expressing (H) scrambled or Rieske siRNA and empty control or Rac1 ${ }_{\mathrm{WT}}$ ( $n$ = 3) and (I) empty, Rac1 ${ }_{C A}$, or Rac1 $1_{D N}$. Arg1 promoter activity in transfected (J) THP-1 cells $(n=3)$ and $(K)$ MH-S cells $(n=3)$. Inset in J shows KLF4 and HIF-2 $\alpha$ immunoblot analysis. mRNA expression of (L) Tgfb1 $(n=3)$ and (M) Chil3 $(n=3)$ in MH-S cells expressing empty control, Rac1 ${ }_{W T}$, or Rac1 ${ }_{\text {c189s. }}$. mRNA expression of $(\mathbf{N}) \operatorname{Tgfb} 1(n=3)$ and $(\mathbf{0})$ Retnla $(n=3)$ in transfected THP-1 cells. Inset in $\mathbf{N}$ shows GGDPS immunoblot analysis. Values indicate the mean \pm SEM. ${ }^{*} P<0.05$, ${ }^{* *} P<0.001$, and ${ }^{* *} P<0.0001$, by 2 -tailed Student's $t$ test (A and $\mathbf{B}$ ) and 1-way ANOVA followed by Tukey's multiple comparisons test (C-E, G, and J-0). Scr, scrambled siRNA.

We questioned whether Rac1 isoprenylation is required to mediate profibrotic macrophage polarization. $\mathrm{MH}-\mathrm{S}$ cells expressing $\mathrm{Rac}_{\mathrm{C} 189 \mathrm{~S}}$ had a significant reduction in the profibrotic genes Tgfb1, Chil3, and Retnla and an increase in proinflammatory genes compared with $\mathrm{MH}-\mathrm{S}$ cells expressing empty control or Rac1 ${ }_{\mathrm{wT}}$ (Figure 4, L and M, and Supplemental Figure 4, F-H). Because GGDPS enzymatically generates the geranylgeranyl moiety for isoprenylation, silencing GGDPS reduced macrophage profibrotic gene expression (Figure 4, N and O) and significantly enhanced $T n f$ and Nos2 (Supplemental Figure 4, I-L). Taken together, these data identify a molecular mechanism by which Rac1-mediated mtROS regulates the switching of macrophages to a profibrotic phenotype.

IPF BAL cells have reduced GGDP levels. To determine the biological relevance of the nonsterol mevalonate pathway and the significance of Rac1 isoprenylation, we determined the concentration of the mevalonate pathway intermediate products (Figure $5 A)$. Mass spectrometry revealed that IPF BAL cells had a 10-fold reduction in farnesyl diphosphate (FDP) and an 11-fold reduction in GGDP compared with BAL cells from healthy volunteers (Figure 5, B-E). Although these products were reduced, the enzymes GGDPS and geranylgeranyltransferase type I (GGTase I) were increased in IPF BAL cells (Figure 5, F-H). Likewise, GGDPS and GGTase I expression was increased in MDMs from mice receiving GGOH or bleomycin compared with macrophages from saline-exposed mice (Figure 5I). These enzymes were further increased in bleomycin-injured mice treated with GGOH (Figure 5 , J and $\mathrm{K}$ ), suggesting that the reduction in GGDP was secondary to an increased flux of isoprenoid equivalents through the nonsterol mevalonate pathway.

GGOH treatment in cells expressing Rac1 ${ }_{\mathrm{wT}}$ led to increased GGDPS and GGTase I expression compared with empty controls (Supplemental Figure 5A). GGDPS expression was markedly increased in Rac1 $1_{\mathrm{C} 189 \mathrm{~s}}$-expressing macrophages, whereas GGTase I expression was essentially absent. We validated these results in vivo. The concentration of GGDP was reduced in bleomycin- injured WT mice, whereas Rac1-/- Lyz2-Cre mice showed increased levels of GGDP regardless of exposure (Supplemental Figure 5B). However, the role of the mevalonate pathway in cholesterol production was not altered by GGOH or bleomycin treatment (Supplemental Figure 5C).

Although the above data suggested an increased flux through the nonsterol mevalonate pathway, the activation of this pathway alone is not known. Because acetyl-CoA is a key precursor of the mevalonate pathway and is a product of $\beta$ oxidation, we sought to determine whether acetyl-CoA was increased in profibrotic macrophages and found that both GGOH and bleomycin increased acetyl-CoA in MDMs from mice (Figure 5L). The cells from bleomycin-injured mice that received GGOH had a higher concentration of acetyl-CoA.

Because Rac1 was required for profibrotic polarization and alternatively activated macrophages reprogram to $\beta$ oxidation for metabolism, we asked whether Rac1 induces $\beta$ oxidation in vivo. MDMs from bleomycin-injured WT mice had a significant increase in the oxygen consumption rate (OCR), whereas macrophages from the Rac1 ${ }^{-/} \mathrm{Lyz2}$-Cre mice had an OCR below that of the saline-exposed control mice (Figure 5M). Moreover, the acetylCoA levels in the macrophages harboring a deletion of Rac1 were significantly lower than the levels detected in WT monocytederived macrophages (Figure $5 \mathrm{~N}$ ). To verify that Rac1 mediated metabolic reprogramming to $\beta$ oxidation, the addition of palmitate to $\mathrm{MH}-\mathrm{S}$ cells expressing constitutively active Rac1 $\left(\mathrm{Rac}_{\mathrm{CA}}\right)$ substantially increased the OCR compared with the BSA control (Figure 5O). In contrast, cells expressing dominant-negative Rac1 $\left(\operatorname{Rac1}_{\mathrm{DN}}\right)$ had a substantial reduction in the maximal OCR to the level of the empty BSA control, with or without palmitate addition.

We questioned whether Rac1 regulates peroxisome proliferator-activated receptor $\gamma$ coactivator-1 $\alpha$ (PGC-1 $\alpha$ ), a transcriptional component of oxidative phosphorylation (OXPHOS) that increases the enzymatic capacity for metabolic reprogramming to $\beta$ oxidation. Rac1 ${ }_{\mathrm{CA}}$ increased nuclear localization of PGC-1 $\alpha$, whereas nuclear PGC-1 $\alpha$ was reduced to levels below the control in Rac1 ${ }_{\mathrm{DN}}$-expressing THP-1 cells (Supplemental Figure 5D). To confirm that the source of acetyl-CoA was from $\beta$ oxidation, we silenced PGC-1 $\alpha$ in THP-1 cells expressing Rac1 $1_{\mathrm{WT}}$ and treated them with GGOH. Both acetyl-CoA (Figure 5P) and Rac1 activation (Figure $5 \mathrm{Q}$ ) were significantly lower in macrophages with silenced PGC-1 $\alpha$, suggesting that acetyl-CoA enhances flux through the mevalonate pathway. Acetyl-CoA was highly correlated with Rac1 activity (Pearson's $r=0.87$ ) (Figure 5R). These data also suggest that a reduction in intermediate products (FDP and GGDP) signifies augmented flux through the pathway required for Rac1 isoprenylation.

Rac1 is required for GGOH- and bleomycin-induced lung fibrosis. Because mitochondrial Rac1 activity is increased in BAL cells from subjects with IPF and GGOH augments mitochondrial Rac1 activity, we asked whether the posttranslational modification is specific for Rac1. We exposed WT and Rac1 ${ }^{-/}$Lyz2-Cre mice to saline or bleomycin. After the initiation of fibrotic repair (10 days after exposure), we administered vehicle or GGOH as described above. No difference was observed in the total number of BAL cells between the mouse strains, and monocytic cells were the predominate cell type (Figure 6, A and B). Rac1 activity was mark- 

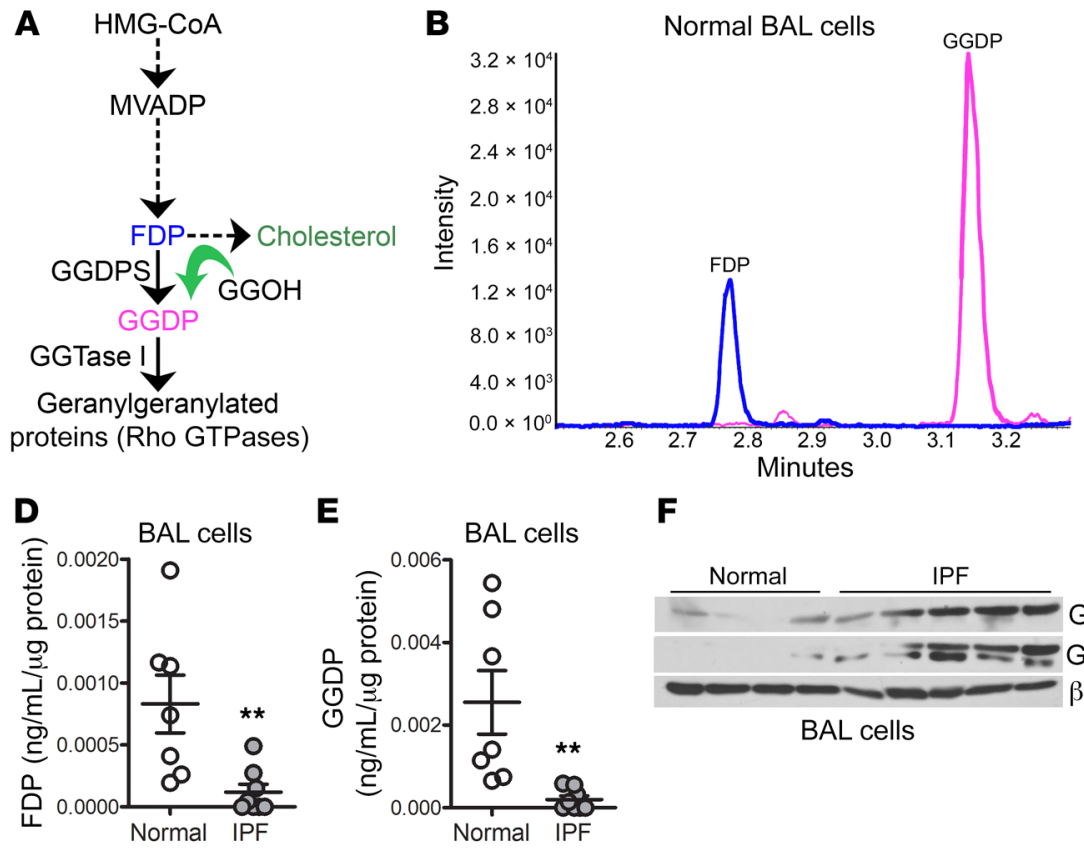

E

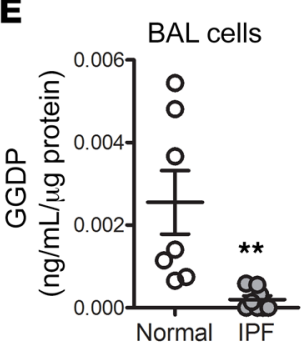

$\mathbf{F}$
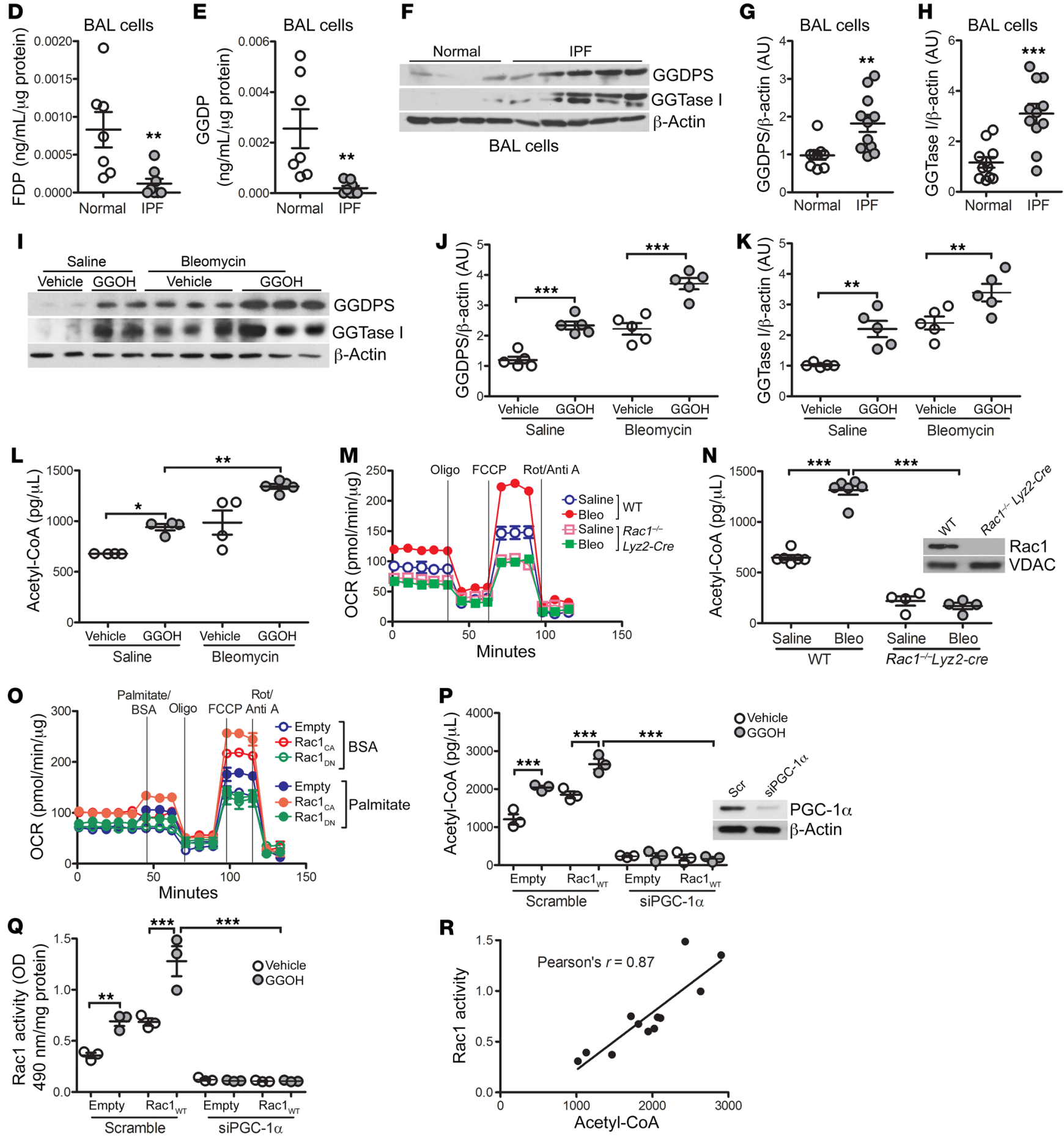
Figure 5. IPF BAL cells have reduced GGDP levels. (A) Schematic diagram of the mevalonate pathway. Representative mass spectrometric chromatography of FDP and GGDP in (B) normal $(n=7)$ and (C) IPF $(n=8)$ BAL cells. Levels of (D) FDP and (E) GGDP in BAL cells from healthy subjects ( $n$ $=7)$ and patients with IPF $(n=8)$ determined by mass spectrometry. $(\mathbf{F})$ Representative immunoblot analysis of BAL cells from healthy subjects $(n=4)$ and patients with IPF $(n=5)$. Quantification of immunoblot in $\mathbf{F}$ of expression of (G) GGDPS and (H) GGTase 1 in BAL cells from healthy subjects ( $n=9-11)$ and patients with IPF $(n=11)$. (I) Representative immunoblot analysis of MDMs from WT mice s.c. treated with vehicle or GGOH 10 days after exposure to saline or bleomycin ( $n=5$ /group). Quantification of (J) GGDPS and (K) GGTase 1 expression in I ( $n=5 /$ group). (L) Acetyl-CoA concentration $(n=4-5)$. (M) OCR and ( $\mathbf{N}$ ) acetyl-CoA concentration in BAL cells isolated from WT or Rac1 ${ }^{-1-}$ Lyz2-Cre mice exposed to saline or bleomycin for 21 days ( $n=5$ mice/group). Inset in $\mathbf{N}$ shows Rac1 immunoblot analysis. (0) OCR in transfected MH-S cells $(n=3-4)$. FCCP, ; Oligo, oligomycin; FCCP, Carbonyl cyanide 4-(trifluoromethoxy)phenylhydrazone; Rot/Anti A, rotenone/antimycin A. (P) Acetyl-CoA concentration and (Q) Rac1 activity in THP-1 cells expressing scrambled siRNA or siPGC-1 $\alpha$ together with empty or Rac1 $1_{W T}$ and treated with GGOH ( $n=3 /$ group). Inset in $\mathbf{P}$ shows PGC-1 $\alpha$ immunoblot analysis. (R) Pearson's correlation of acetyl-CoA to Rac1 activity. Values indicate the mean \pm SEM. ${ }^{*} P<0.05,{ }^{* *} P<0.001$, and ${ }^{* *} P<0.0001$, by 2-tailed Student's $t$ test statistical analysis (D, E, G, and $\mathbf{H}$ ), 1-way ANOVA followed by Tukey's multiple comparisons test (J-L, $\mathbf{N}, \mathbf{P}$, and $\mathbf{Q})$, and Pearson's correlation analysis (R).

edly reduced in BAL cells from $\mathrm{Rac1}^{-/-} \mathrm{Lyz2}$-Cre mice compared with WT BAL cells, and neither GGOH nor bleomycin altered the activity (Figure 6C). The deletion of Rac1 in macrophages did not alter Rac1 expression in AECs or neutrophils (Supplemental Figure 6, A and B). Furthermore, the activity of Rac2 was not altered by bleomycin exposure or GGOH treatment in either strain (Supplemental Figure 6C). We found that the absence of mitochondrial Rac1 activity resulted in a significant reduction of mtROS generation in BAL cells from $\mathrm{Racl}^{-1-} \mathrm{Lyz2}$-Cre mice, regardless of exposure (Figure 6D). Moreover, the levels of active TGF- 31 , PDGF-BB, and $\mathrm{Ym}-1$ in BALF from $\mathrm{Racl}^{-/-} \mathrm{Lyz2}$-Cre mice were significantly reduced to levels below those in WT saline-treated control mice (Figure 6, E-G).

The role of statins in the development of interstitial lung disease remains controversial. We sought to determine whether simvastatin treatment after the onset of fibrosis (10 days after bleomycin injury) has an impact on fibrotic repair. We found that simvastatin treatment promoted Rac1 activity in monocytic cells and that the combination of simvastatin and bleomycin exposure further significantly increased Rac1 activity (Supplemental Figure 6D). Because Akt is linked to fibrosis development and statins activate Akt $(9,31,32)$, Rac1 activity was significantly reduced in mice with conditional deletion of Akt1 in MDMs (Akt1 ${ }^{-/-}$Lyz2-Cre). Furthermore, simvastatin promoted Akt1 activation in MDMs from WT mice, an effect that was further increased with simvastatin and bleomycin together (Supplemental Figure 6E). Simvastatin alone increased active TGF- $\beta 1$ and PDGF-BB levels in BALF, and these levels were further increased in bleomycin-injured WT mice (Figure 6, H and I). The biological significance of these findings revealed that simvastatin treatment alone mediated lung fibrosis, and mice with bleomycin-induced fibrosis showed significantly increased hydroxyproline levels when treated with simvastatin (Supplemental Figure 6F). In contrast, $A k t 1^{-/-}$Lyz2-Cre mice were protected from bleomycin- and simvastatin-mediated fibrosis.
Extending these observations, the deletion of Rac1 in macrophages protected mice from bleomycin-induced fibrosis as well as from GGOH-mediated fibrosis, whereas GGOH or bleomycin treatment increased collagen deposition in WT mice (Figure 6J). As expected, administration of GGOH to bleomycin-injured mice exacerbated collagen deposition to a greater extent. These findings were confirmed by measuring lung hydroxyproline content (Figure 6K). Taken together, these data suggest that the posttranslational modification of Rac1 in MDMs is a critical regulator of fibrotic repair.

Fibroblast differentiation and collagen production are macrophage dependent. To further define the role of MDM/fibroblast signaling, we generated pulmospheres (3D spheroids composed of cells derived from lung tissue in which all lung cell types are present in situ) from mice, as recent evidence suggests that pulmospheres from the lungs of patients with IPF permit a personalized approach to therapy through evaluation of the zone of invasion (ZOI) of myofibroblasts (33). MDMs were removed from the cell suspension with CD11b and Ly6C antibodies (Figure 7A). We observed that the ZOI was significantly increased in pulmospheres isolated from GGOH-treated mice when macrophages were present, and pulmospheres from bleomycin-injured mice showed a similar effect (Figure 7, B and C). The ZOI was further significantly increased in pulmospheres isolated from bleomycin-injured mice treated with GGOH with macrophages intact compared with bleomycin-injured mice; however, the ZOI was mitigated when macrophages were removed in all conditions.

The differentiation of fibroblasts into myofibroblasts plays a critical role in fibrosis, and macrophage-derived TGF- $\beta 1$ production is critical for fibroblast differentiation (9). IMR-90 fibroblasts incubated with BALF from bleomycin-injured mice or GGOH-treated mice showed an increase in $\alpha$-smooth muscle actin ( $\alpha$-SMA), and the combination induced increased differentiation into myofibroblasts (Figure 7D). Expression of $\alpha$-SMA was absent in fibroblasts incubated with BALF from Rac1 ${ }^{-/}$Lyz2-Cre mice. Because RhoA is required for fibroblast differentiation (34), RhoA activity in IMR90 fibroblasts paralleled $\alpha$-SMA expression in fibroblasts, whereas RhoA activity was significantly reduced in fibroblasts incubated with BALF from $\mathrm{Rac1}^{-/}$Lyz2-Cre mice (Figure 7E).

We questioned whether the increase in fibroblast RhoA activity was due to GGOH treatment as seen with Rac1 in macrophages. Primary fibroblasts isolated from GGOH-treated mice had a significant increase in RhoA activity compared with fibroblasts from mice that received vehicle treatment (Figure 7F). In IMR-90 fibroblasts treated with GGOH in vitro, we observed no effect on RhoA activity compared with the vehicle-treated cells (Figure 7G). These results suggest that the posttranslational modification of RhoA is being regulated differently than the mechanism observed in macrophages.

Because RhoA activity was increased in primary fibroblasts from mice and TGF- $\beta 1$ was increased in BALF, we tested whether macrophage-derived TGF- $\beta 1$ was responsible for increasing RhoA activity in fibroblasts. MH-S cells were treated with GGOH, and IMR-90 fibroblasts were cultured in the conditioned media in the presence or absence of a TGF- $\beta 1$-neutralizing antibody. Conditioned media from the GGOH-treated macrophages significantly increased RhoA activity (Figure $7 \mathrm{H}$ ); however, these changes were reversed by neutralizing TGF- $\beta 1$. IMR-90 fibroblast differentiation 

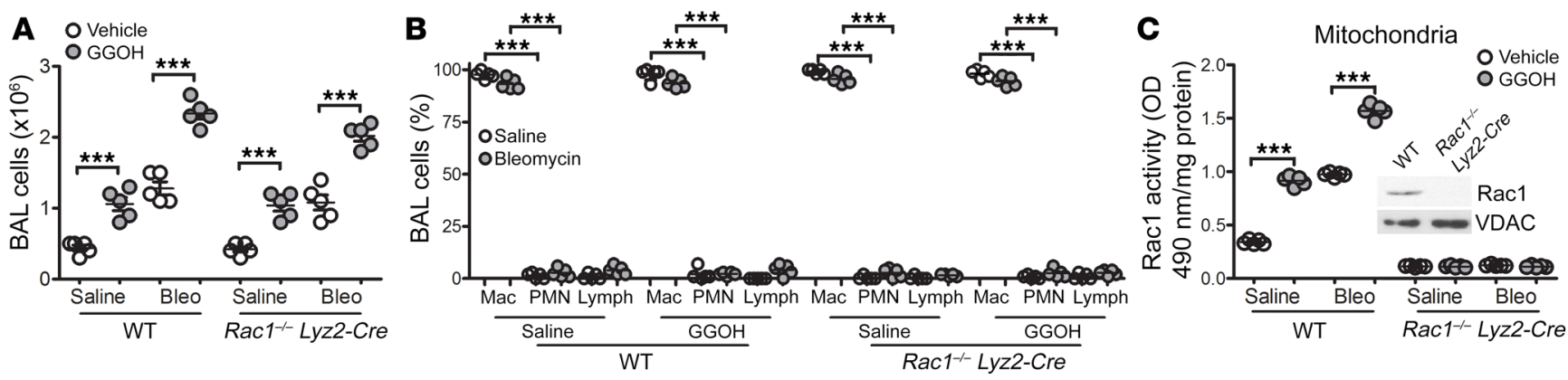

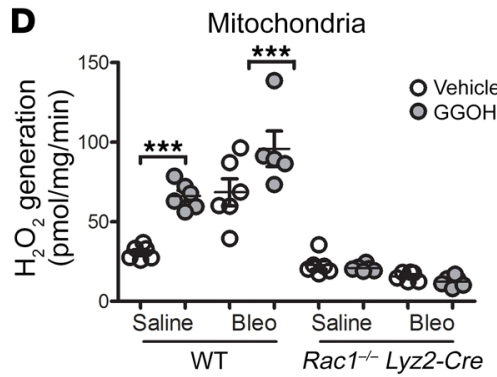

E

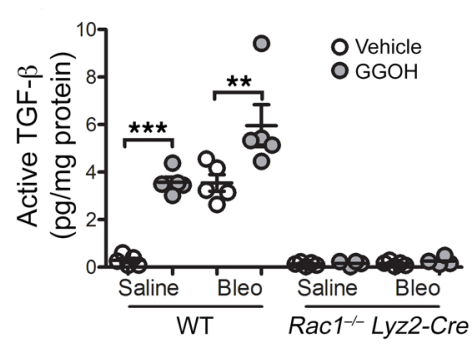

H
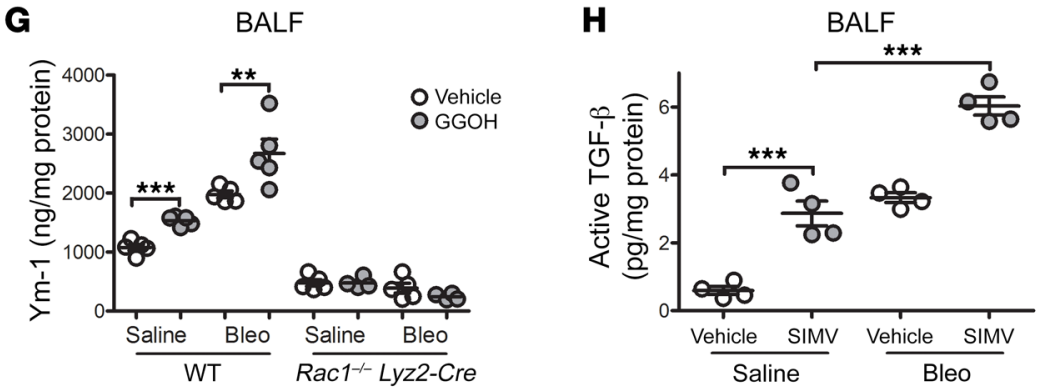

WT

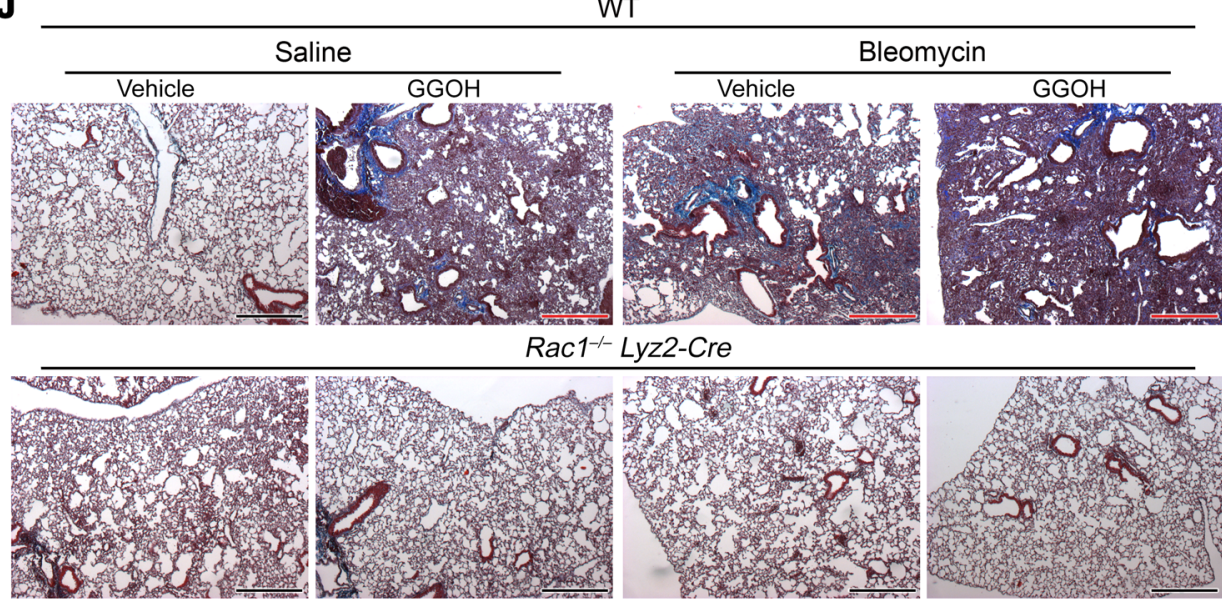

$\mathbf{F}$

BALF

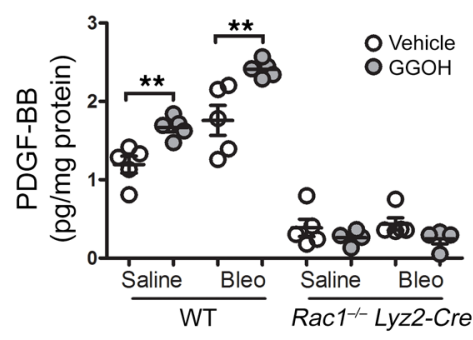

I

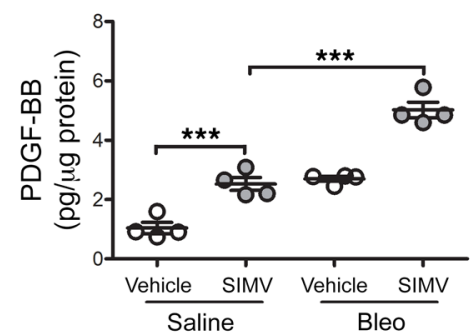

$\mathbf{K}$

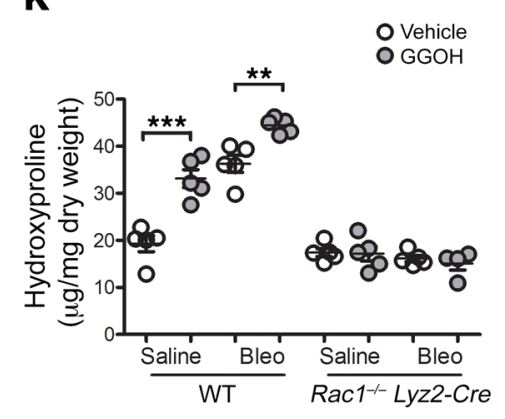

Figure 6. Rac1 is required for GGOH- and bleomycin-induced lung fibrosis. Ten days after exposure of WT and Rac1 ${ }^{1-}$ Lyz2-Cre mice to saline or bleomycin, pumps containing vehicle or GGOH were s.c. implanted, and the mice were sacrificed 11 days later. (A) Total number of BAL cells and (B) cell differential ( $n$ = 5/group). Mac, macrophage; PMN, polymorphonuclear. (C) Mitochondrial Rac1 activity ( $n=5$ /group). Inset in C shows Rac1 immunoblot analysis of BAL cells. (D) mtROS generation in isolated BAL cells ( $n=5$-6/group). (E) Active TGF- $\beta 1$, (F) PDGF-BB, and (G) Ym- 1 expression in BALF ( $n=4-5 / g r o u p)$. (H and I) Ten days after exposure of WT mice to saline or bleomycin, daily i.p. injections of simvastatin (SIMV) (20 mg/kg/day) were administered, and mice were sacrificed 11 days later. (H) Active TGF- $\beta 1$ and (I) PDGF-BB expression in BALF ( $n=4 /$ group). (J) Representative images of lung histology with Masson's trichrome staining ( $n=5$ /group). Original magnification, $\times 2.5$. (K) Hydroxyproline content $\left(n=5 /\right.$ group). Values indicate the mean \pm SEM. ${ }^{* *} P<0.001$ and ${ }^{* * *} P<0.0001$, by 1-way ANOVA followed by Tukey's multiple comparisons test. 
corresponded to the RhoA activity, as neutralizing TGF- $\beta 1$ suppressed fibroblast differentiation (Figure 7I). Likewise, fibroblast Fn1 and Col1a1 mRNA expression was significantly reduced when these cells were cultured in TGF- $\beta 1$-neutralized conditioned media (Figure 7, J and K). Similar findings were obtained in vivo (Figure 7, $\mathrm{L}$ and $\mathrm{M})$. These data suggest that enhanced Rac1 isoprenylation mediates MDM/fibroblast signaling, in that RhoA activity is dependent on macrophage-derived TGF- $\beta 1$ production and is required to promote fibroblast differentiation. Moreover, these observations provide a molecular mechanism or mechanisms by which MDMs orchestrate fibrotic repair in the absence of lung injury and identify a therapeutic target for attenuation of fibrotic repair.

\section{Discussion}

Pulmonary fibrosis is an aberrant response to lung injury that involves several cell types. The major cell types that participate in responses to lung injury include epithelial cells, fibroblasts, and myeloid-derived immune cells. The existing paradigms of pulmonary fibrosis have focused on abnormal epithelium-fibroblast interactions $(5,6,23)$. Here, we show a paradigm shift based on emerging information that indicates a critical and essential role for MDM-fibroblast crosstalk in the development and progression of fibrosis in the absence of epithelial injury.

The mevalonate pathway is a therapeutic target in multiple diseases (35-37). Although statins can reduce the activation of Rac1 and RhoA, the relationship between statin usage and the incidence of interstitial lung disease is controversial. Statin use has been shown to attenuate lung function decline in aging men (38). A recent post hoc analysis of subjects with IPF who were receiving placebo in the CAPACITY and ASCEND trials shows that statin users had reduced hospitalization and mortality compared with those who did not use statins; however, no significant difference was detected in forced vital capacity (FVC) decline or disease progression (39). Results obtained in a post hoc analysis of pooled data from the INPULSIS trials did not establish a beneficial effect of statin use in patients with IPF (40), with similar results shown by others $(41,42)$. None of these studies evaluated the effect of statins on the activation of Ras or Rho GTPases. We found that statins promoted pulmonary fibrosis via Akt1-mediated activation of Rac1 in macrophages. Moreover, we previously showed that Akt1 increased flux through the mevalonate pathway, which resulted in Rac1 activation (31). A review from the US FDA reported an association of statin use and development of interstitial lung diseases, with development of pulmonary fibrosis constituting up to $0.4 \%$ of the adverse events (43). Furthermore, statin use is associated with interstitial lung abnormalities in individuals who smoke, and statins increase the degree of fibrosis in bleomycin-exposed mice (44).

In osteoporosis, $\mathrm{N}$-bisphosphonates adsorb to bone mineral to reduce bone resorption by inhibiting farnesyl diphosphate synthase (15). Inhibitors of farnesyl transferase and GGTase I, which catalyze the transfer of farnesyl and geranylgeranyl moieties to Ras and Rho GTPases, respectively, have been used in cancer therapy because Ras and Rho GTPases are essential for cell growth, proliferation, and apoptosis resistance $(17,37)$. The mevalonate pathway, however, has not, to our knowledge, been investigated in pulmonary fibrosis. By supplying more substrate for geranylgeranylation (GGOH), Rac1 activation was increased, resulting in the polarization of macrophages to a profibrotic phenotype. This polarization resulted in a robust enhancement of fibroblast differentiation and collagen deposition, suggesting that inhibition of the nonsterol mevalonate pathway specifically may be a therapeutic strategy for treating a chronic and progressive disease such as IPF.

The Rho GTPases have a critical role in host defense. One member of these GTP-binding proteins, Rac1, is known to regulate assembly of the actin cytoskeleton, activation of NOX2 in nonphagocytic cells, and migration, adhesion, and differentiation in certain cell types $(45,46)$. Rac1 mediates ROS generation in many disease processes $(22,25,45,47)$, and our prior work demonstrated that import of Rac1 into the mitochondrial intermembrane space is required for mtROS generation in macrophages (22). These functions of Rac1 require a posttranslational modification - geranylgeranylation - of the C-terminal cysteine residue $\left(\mathrm{Cys}^{189}\right)$ for activation and interaction with other proteins (48). The association of Rac1 or other Rho GTPases with the macrophage phenotype is not known; however, the generation of mtROS is a Th2-independent mechanism of profibrotic polarization $(8,9,13,14)$. Moreover, IPF BAL cells display apoptosis resistance while promoting disease progression as a result of their increased production of TGF- $\beta 1$ (9). Taken together, the current observations indicate that augmenting the posttranslational modification of Rac1 enhances mtROS to induce the profibrotic phenotypic switching of macrophages.

A long-standing dogma holds that AEC injury and subsequent apoptosis are required for the development of lung fibrosis $(5,6,23)$. There is also evidence that some AECs undergo several biological changes to assume a mesenchymal cell phenotype, which induces a migratory capacity with invasiveness, apoptosis resistance, and increased production of extracellular matrix (49). TGF- $\beta 1$ has been implicated in the induction of AEC apoptosis, with the exception of when cells are attached to matrix components, in which case TGF- $\beta 1$ initiates mesenchymal transition (6). Our data show that enhancement of Rac1 activation with GGOH does not induce AEC injury or apoptosis; however, macrophage-derived TGF- $\beta 1$ production and activation are increased to mediate fibrosis. These observations support the notion that the heightened posttranslational modification of Rac1 mediates AEC stability rather than injury and apoptosis.

Fibroblasts are the final effector cells in fibrosis. RhoA is known to be critical for fibrosis in multiple organs (50-52). Although RhoA is known to be necessary for the differentiation of fibroblasts, the mechanism by which RhoA is activated in fibroblasts has not been clearly defined. We recently showed that mice with a conditional deletion of TGF- $\beta 1$ in lung macrophages are protected from pulmonary fibrosis (9). In addition, the inability to activate latent TGF- $\beta 1$ in $\alpha v \beta 6^{-/-}$mice attenuated bleomycin-induced pulmonary fibrosis (53). The current data complement our findings, as MDMs are a critical source of TGF- $\beta 1$ in the lungs (9), and pulmospheres lacking MDMs have reduced fibroblast invasion. In aggregate, these observations implicate a shift in the current paradigm. Although AEC dysfunction may initiate the development of $\operatorname{IPF}(5,6,23)$, our findings suggest that repeated AEC injury is not required for the progression of fibrosis. Furthermore, we propose 
A
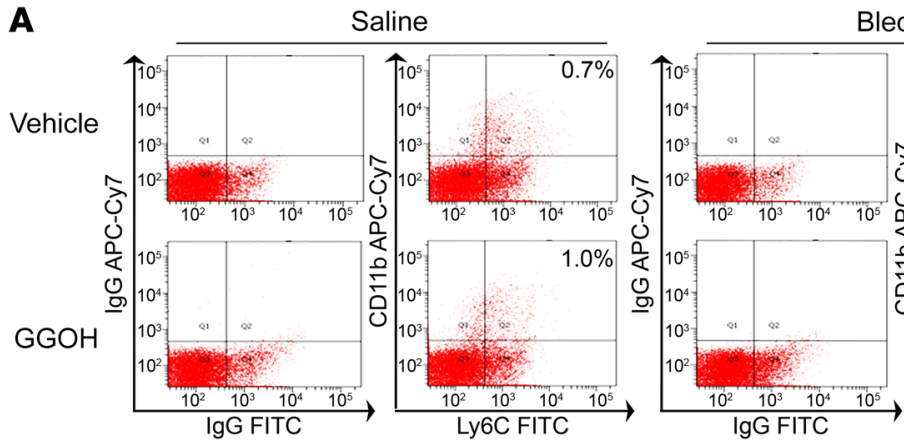

Bleomycin

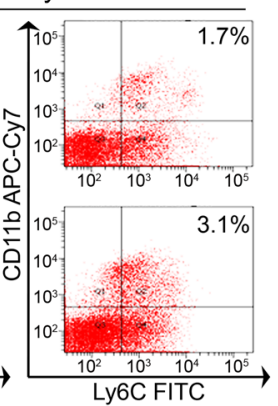

B

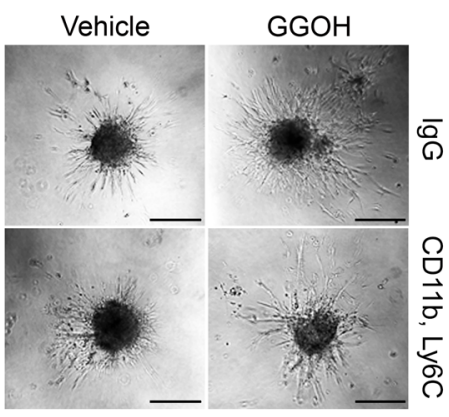

C

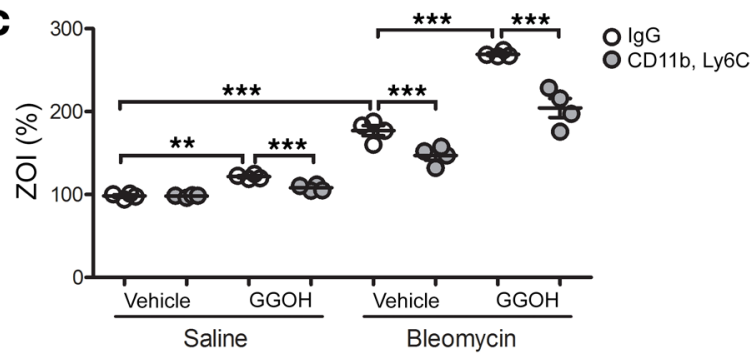

D

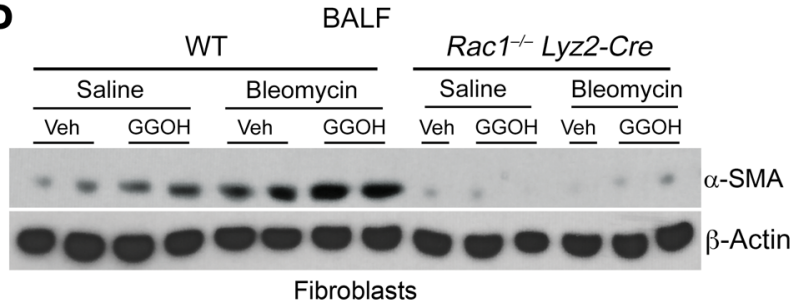

E

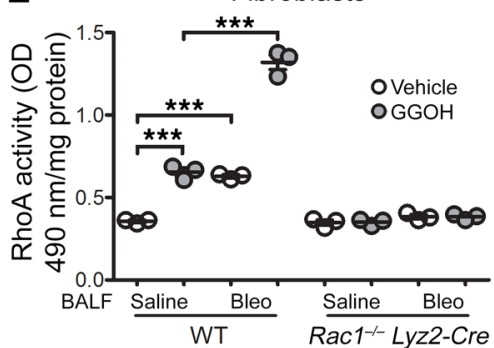

H

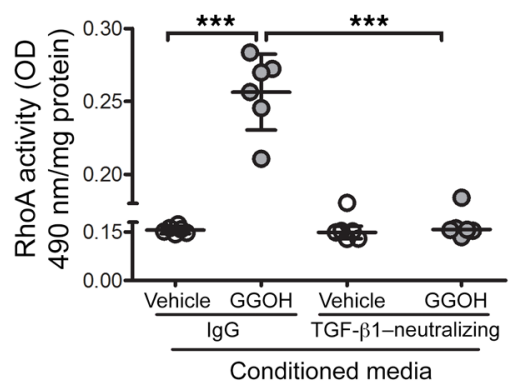

K

Fibroblasts

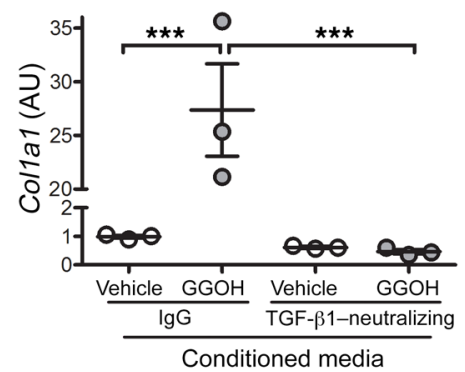

$\mathbf{F}$

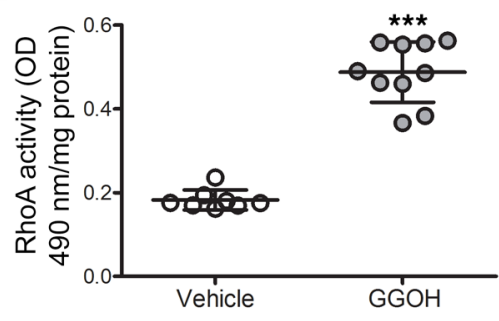

I

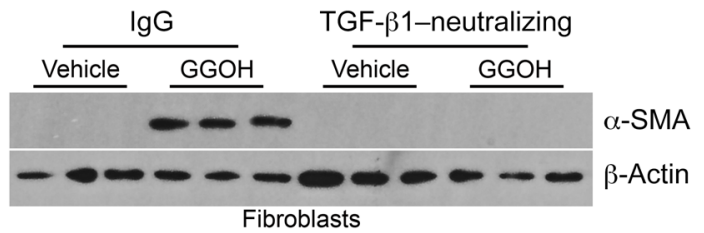

Fibroblasts
G
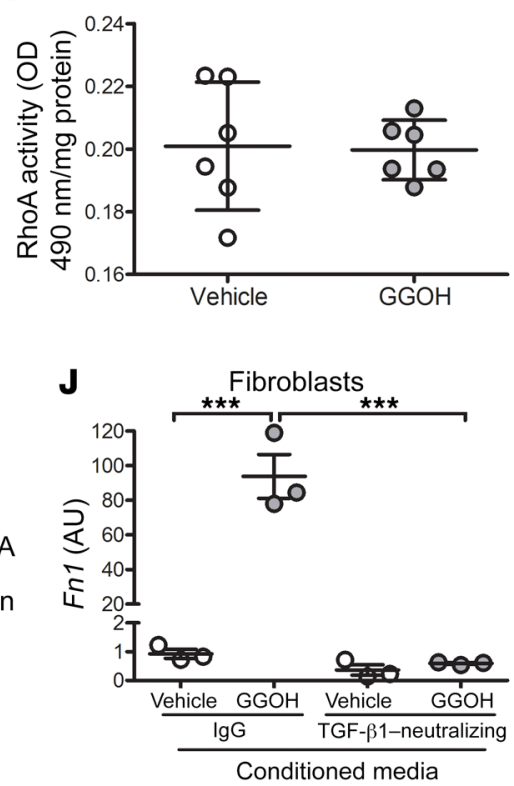

L

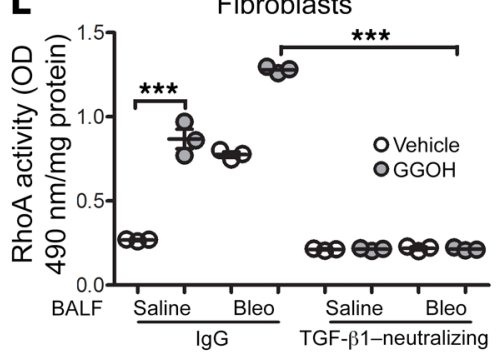

M

BALF

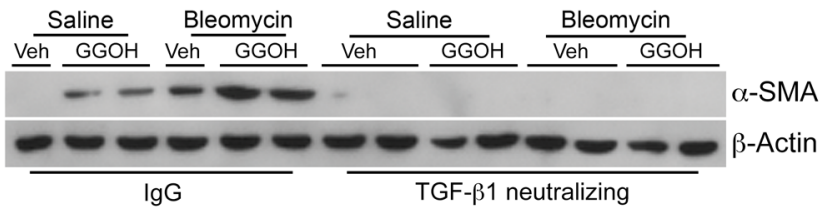


Figure 7. Fibroblast differentiation and collagen production are macrophage dependent. Ten days after exposure of WT mice to saline or bleomycin, pumps containing vehicle or $\mathrm{GGOH}$ were s.c. implanted, and lungs were extracted 11 days later. Cell suspensions from WT mice were incubated with IgC or CD11b and Ly6C antibodies. (A) Representative histograms of sorted cells ( $n=4$ /group). The percentage indicates cells removed for each condition. (B) Representative phase contrast microscopy pulmosphere images after a 16 -hour incubation ( $n=4 /$ group). Scale bars: $200 \mu \mathrm{m}$. (C) Percentage of the ZOI ( $n=4 /$ group). ZOI percentage $=[$ (total area after invasion - inner core of pulmosphere)/inner core of pulmosphere] $\times 100$. (D) Immunoblot analysis of fibroblasts cultured in BALF from exposed WT and Rac1-- Lyz2-Cre mice ( $n=2-3$ mice/group). (E) RhoA activity in fibroblasts cultured in BALF from exposed WT and Rac1-1- Lyz2-Cre mice ( $n=3$ mice/group). (F) RhoA activity in primary mouse lung fibroblasts isolated from vehicle- or GGOH-treated mice $(n=$ 8-10). (G) RhoA activity in IMR-90 fibroblasts $(n=3)$. IMR-90 fibroblasts were cultured in conditioned media from vehicle- or $\mathrm{GGOH}$-treated $\mathrm{MH}-\mathrm{S}$ cells containing IgG control or TGF- $\beta 1$-neutralizing antibody. (H) RhoA activity in IMR-90 fibroblasts ( $n=6 /$ group). (I) Immunoblot analysis in IMR-90 fibroblasts. mRNA analysis of (J) Fn1 ( $n=3 /$ group) and (K) Col1a1 mRNA in IMR-90s ( $n=3 /$ group). IMR-90 fibroblasts cultured in BALF from saline- or bleomycin-exposed mice that received vehicle or $\mathrm{GCOH}$. BALF was preincubated with IgG or neutralized with a TGF- $\beta 1$ antibody. (L) RhoA activity in IMR-90 fibroblasts ( $n=3$ /group). (M) Immunoblot analysis of IMR-90 fibroblasts. Values indicate the mean \pm SEM. ${ }^{* *} P<$ 0.001 and ${ }^{* * *} P<0.0001$, by 1-way ANOVA followed by Tukey's multiple comparisons test (C, E, $\mathbf{H}$, and $\mathbf{J}-\mathbf{L}$ ) and 2-tailed $t$ test ( $\mathbf{F}$ and $\mathbf{G}$ ).

that MDM-fibroblast crosstalk can induce fibrosis without AEC injury and is the primary driver in mediating disease progression.

\section{Methods}

Human subjects. We obtained BAL cells from healthy subjects and patients with IPF. Healthy volunteers had to meet the following criteria: (a) age between 18 and 65 years; (b) no history of cardiopulmonary disease or other chronic disease; (c) no prescription or nonprescription medication except oral contraceptives; (d) no recent or current evidence of infection; and (e) lifetime nonsmoker. Patients with IPF had to meet the following criteria: (a) FVC of at least $50 \%$ predicted; (b) current nonsmoker; (c) no recent or current evidence of infection; and (d) evidence of restrictive physiology on pulmonary function tests and usual interstitial pneumonia on chest CT. Fiberoptic bronchoscopy with BAL was performed after the subjects received local anesthesia. Three subsegments of the lung were lavaged with five 20-mL aliquots of normal saline, and the first aliquot in each was discarded. The percentage of macrophages was determined by Wright-Giemsa staining and varied from $90 \%$ to $98 \%$.

Mice. Rac1 ${ }^{-1-}$ Lyz2-Cre and Akt1 ${ }^{-/}$Lyz2-Cre, mice were generated by selective disruption of the Rac1 or Akt1 gene in the cells of the granulocyte/monocyte lineage as previously described $(9,54)$. Eight- to 12-week-old male and female C57/BL6 WT, Rac1 ${ }^{-/} \mathrm{Lyz2-Cre,} \mathrm{Akt1}^{-1}$ Lyz2-Cre, CCR2 $2^{--}$, and littermate control mice were intratracheally administered $1.75 \mathrm{U} / \mathrm{kg}$ bleomycin, $125 \mu \mathrm{g}$ chrysotile asbestos suspension, or saline or $\mathrm{TiO}_{2}$ as a negative control, after anesthetization with $3 \%$ isoflurane using a precision Fortec vaporizer. Ten days after exposure, miniosmotic pumps (no. 1004, Durect) containing either vehicle (polyethylene glycol) or GGOH (300 mg/kg/day, MilliporeSigma) were implanted s.c. as described previously (55). Mice were anesthetized with ketamine $(60 \mathrm{mg} / \mathrm{kg})$ and xylazine $(12.5 \mathrm{mg} / \mathrm{kg})$, and a small incision was made in the skin on the upper back. Pumps were inserted and the skin was stapled closed. Mice were treated with simvastatin $(20 \mathrm{mg} / \mathrm{kg} /$ day, MilliporeSigma) or saline i.p. daily beginning 10 days after exposure to bleomycin or saline. BAL was harvested by cannulating the trachea and performing total lavage of the lungs with sterile saline using the flow of gravity to collect cells and fluid. AECs were isolated as previously described (56).

Cell culturing. Human monocyte (THP-1), mouse alveolar macrophage (MH-S), and IMR-90 normal lung fibroblast cell lines were obtained from the American Type Culture Collection (ATCC). Macrophages were maintained in RPMI 1640 media with $10 \%$ FBS and penicillin-streptomycin supplements. IMR-90 fibroblasts were cultured in DMEM supplemented with $10 \%$ FBS, streptomycin, and amphotericin D. All experiments were conducted in RPMI containing 0.5\% serum. In select experiments, macrophages were treated with $50 \mu \mathrm{M}$ GGOH or vehicle overnight.

Quantitative real-time PCR. Total RNA was isolated and reverse transcribed, and quantitative real-time PCR was performed as described previously (9). The following primer sets were used: human arginase-1 (5'-TTCTCAAAGGGACAGCCACG-3' and 5'-TAGGGATGTCAGCAAAGGGC-3'); human PDGF-B (5'-AGCCTCGCTGCAAAG AGAAA-3' and 5' ${ }^{\prime}$-ATCTTCCTCTCCGGGGTCTC- $3^{\prime}$ ); mouse PDGF-B (5'-CTGCCCCTCAAAAGCCTAGT- ${ }^{\prime}$ and $5^{\prime}$-TGACCACCATCCCTGTGAAAT- $3^{\prime}$ ); and mouse TGF- $\beta 1$ ( $5^{\prime}$-CGGAGAGCCCTGGATACCA-3' and 5'-TGCCGCACA CAGCAGTTC-3'). Data were calculated by the ${ }^{\Delta \Delta} \mathrm{Ct}$ method, normalized to $\beta$-actin or HPRT, and expressed in AU.

Liquid chromatography-mass spectrometry of FDP and GGDP. Samples were precipitated with cold methanol/75 $\mathrm{mM} \mathrm{NH}_{4} \mathrm{OH}$ /isopropanol (1:1.5:5). The supernatant was dried under $\mathrm{N}_{2}$ gas and reconstituted in $10 \%$ methanol to match LC-MS gradient starting conditions. Samples were chromatographically separated using the Prominence 20AD LC system (Shimadzu) over a Zorbax Extend $\mathrm{C}_{18}$ column $(5 \mu \mathrm{m}, 150 \times$ $2.1 \mathrm{~mm}$ ) at $4^{\circ} \mathrm{C}$. Mobile phases were A: $10 \mathrm{mM} \mathrm{NH}_{4} \mathrm{OH}$ (pH 10.5) and B: $70 \%$ acetonitrile/ $30 \%$ isopropanol/0.1\% $\mathrm{NH}_{4} \mathrm{OH}$ at a flow rate of 0.5 $\mathrm{mL} / \mathrm{min}$. A binary gradient starting at $10 \% \mathrm{~B}$ was linearly increased to $95 \%$ B at 4 minutes; then $95 \%$ B was held for 0.5 minutes to wash the column. From 4.5 to 4.75 minutes, B was lowered to $10 \%$, and the column was equilibrated in $10 \% \mathrm{~B}$ for 5.25 minutes. The preinjection and postinjection needle wash was $50 \%$ methanol. Column eluent was diverted to waste from 0 to 0.5 minutes and then again from 4.5 to 10 minutes. The total analysis time was $10 \mathrm{~min} / \mathrm{sample}$, and injection volumes were $20 \mu \mathrm{l}$. Multiple reaction monitoring was carried out on a 6500 QTRAP (SCIEX) mass spectrometer running Analyst 1.6.2 (SCIEX), operating in negative polarity electrospray ionization mode. Mass spectrometric parameters were: IS-4300 v, TEM 600, GS1 60, GS2 60, CUR 35, and CAD medium. Mass transitions monitored for FDP and GGDP were $m / z 381 / 79$ and 449/79, respectively. The compound-specific parameters were: CXP-13, DP-45, and EP-10. Collision energy (CE) for FDP was 40, and CE for GGDP was 46. Postacquisition data analysis was performed using MultiQuant 3.0.1 (SCIEX). Calibration curve regressions were linear, with $1 / x^{2}$ weighting over the range from 0.5 to $100 \mathrm{ng} / \mathrm{mL}$. The limit of detection was $0.25 \mathrm{ng} / \mathrm{mL}$.

Plasmids, transfections, and siRNA. The pRK-Flag-Rac1 ${ }_{\mathrm{wT}}$ and pRK-Flag-Rac1 ${ }_{\mathrm{C} 189 \mathrm{~s}}$ plasmids have been previously described (22). The mouse Arg1 promoter/enhancer (Addgene, 34571) and the Retnla (NC_000082.6) promoter gene were amplified from total genomic DNA. The PCR product was digested with XhoI and HindIII and ligat- 
ed into the pGL3-Basic vector. The correct reading frame and sequence were verified by the UAB Heflin Center Genomics Core. Promoter gene expression was evaluated using luciferase reporter plasmids. Cells were transfected using X-treme GENE 9 Transfection Reagent (MilliporeSigma) according to the manufacturer's protocol. Renilla and firefly luciferase activity was determined in cell lysates using the Dual Luciferase Reporter Assay Kit (Promega) and normalized to the firefly control. Cells were transfected with $100 \mathrm{nM}$ scrambled siRNA, human Rieske, KLF4, HIF-2 $\alpha$, or GGDPS siRNA duplex (IDT) using Dharmafect 2 or Duo (Dharmacon) according to the manufacturer's protocol. Eight hours after transfection, media were replaced and cells were allowed to recover for 24 to 72 hours.

Confocal imaging. BAL cells were fixed with $4 \%$ paraformaldehyde in PBS for 45 minutes at room temperature, permeabilized for 3 minutes, and stained with antibodies in PBS containing 5\% BSA for 45 minutes. Hamster anti-CD11c-FITC (117305; BioLegend) or rat antiCD11b (101201; BioLegend) and goat anti-rat IgG-FITC (3030-02; Southern Biotech) antibodies were used, and DAPI was used for counterstaining. The Nikon A1 confocal microscope was used for imaging.

Flow cytometry. BAL cells were blocked with 1\% BSA containing TruStain fcX anti-mouse CD16/32 antibody (101319; BioLegend), followed by staining with antibodies. The following antibodies were used: rat anti-mouse CD45-PE (12-0451-82; eBiosciences); Live/ Dead eFluor 506 (65-0866; Invitrogen, Thermo Fisher Scientific); rat anti-mouse CD11b-APC-Cy7 (101225; BioLegend); anti-mouse CD64-PE-Cy7 (139313; BioLegend); rat anti-mouse Ly6G-AF700 (561236; BD); rat anti-mouse Siglec F-APC (155507; BioLegend); rat anti-mouse Ly6C, eFluor 450 (48-5932-82; Invitrogen, Thermo Fisher Scientific); Armenian hamster anti-mouse CD11c-FITC (117305; BioLegend); and rat anti-mouse MHC II-PerCP-Cy5.5 (562363; $\mathrm{BD})$. A hierarchical gating strategy was used to represent the RAM

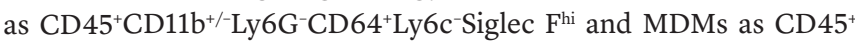
$\mathrm{CD}_{11 \mathrm{~b}^{+/}{ }^{+} \text {Ly6G }}{ }^{-} \mathrm{CD} 64^{+}$Ly6c $\mathrm{c}^{-}$Siglec $\mathrm{F}^{\mathrm{lo}}$. Data were acquired on an LSR II (BD Biosciences) using BD FACSDiva software, version 8.0.1. Data were analyzed with FlowJo software, version 10.5.0.

Pulmosphere invasion. Pulmosphere preparation was performed as previously described (33). Single-cell suspensions of mouse lungs were obtained by collagenase digestion and filtered through 100$\mu \mathrm{m}$ cell strainers, and RBCs were lysed. Cells $\left(5 \times 10^{6}\right)$ were stained with the appropriate antibody (anti-mouse CD11b FITC, anti-mouse Ly6C APC-Cy7, both from BioLegend) in FACS buffer. IgG-FITC and IgG-APC-Cy7 isotype antibodies were used for controls. The doublenegative cells (FITC ${ }^{-}, \mathrm{APC}^{-\mathrm{Cy}^{-}}$) from samples incubated with isotype controls and the $\mathrm{CD} 1 \mathrm{~b}^{-}$and $\mathrm{Ly} \mathrm{C}^{-}$cells were sorted on a $\mathrm{BD}$ FACSAria. Cells were used to prepare pulmospheres in HEMA coated plates. Pulmospheres were seeded in collagen matrix and incubated for 16 hours. The invasiveness was calculated as a percentage of the ZOI: ZOI percentage $=[$ (total area after invasion - inner core of pulmosphere)/inner core of pulmosphere] $\times 100$.

Tissue digestion. Lung, kidney, and liver tissue was harvested from mice and digested. Lung tissue was digested in HBSS with $\mathrm{Mg}^{2+}$ and $\mathrm{Ca}^{2+}$ containing $0.2 \mathrm{mg} / \mathrm{mL}$ DNase I and $2 \mathrm{mg} / \mathrm{mL}$ collagenase D, kidney tissue was digested in RPMI 1640 containing $1 \mathrm{mg} / \mathrm{mL}$ collagenase type I and $100 \mathrm{U} / \mathrm{mL}$ DNase I, and liver tissue was digested in RPMI containing $0.5 \mathrm{mg} / \mathrm{mL}$ collagenase with $100 \mathrm{U} / \mathrm{mL}$ DNase type I for 30 minutes at $37^{\circ} \mathrm{C}$. Tissue fragments were passed through a $70-\mu \mathrm{m}$ mesh, which yielded single-cell suspensions. RBCs were lysed, and cells were resuspended in FACS buffer containing 1\% BSA with Fc blocking solution for 30 minutes on ice. Cells were stained for $30 \mathrm{~min}$ utes at room temperature with a primary antibody and then washed and resuspended in FACS staining buffer.

Determination of $\mathrm{H}_{2} \mathrm{O}_{2}$ generation. $\mathrm{H}_{2} \mathrm{O}_{2}$ production was determined by fluorometry. Cells were incubated in phenol red-free HBSS supplemented with $6.5 \mathrm{mM}$ glucose, $1 \mathrm{mM}$ HEPES, $6 \mathrm{mM}$ sodium bicarbonate, $1.6 \mathrm{mM}$ pHPA, and $0.95 \mu \mathrm{g} / \mathrm{mL}$ HRP. Fluorescence of the pHPA dimer was measured using a spectrofluorometer at an excitation of $320 \mathrm{~nm}$ and an emission of $400 \mathrm{~nm}$ (9).

Isolation of mitochondria and cytoplasm. Mitochondria and cytoplasm were isolated as previously described (9).

Nuclear isolation. Nuclear isolation was performed by resuspending cells in a lysis buffer (10 mM HEPES, $10 \mathrm{mM} \mathrm{KCl,} 2 \mathrm{mM} \mathrm{MgCl}$, 2 mM EDTA) for 15 minutes on ice. Nonidet P40 (10\%) was added to lyse the cells, and the cells were centrifuged at $4^{\circ} \mathrm{C}$ at $20,000 \mathrm{~g}$. The nuclear pellet was resuspended in an extraction buffer $(50 \mathrm{mM}$ HEPES, $50 \mathrm{mM} \mathrm{KCl}, 300 \mathrm{mM} \mathrm{NaCl}, 0.1 \mathrm{mM}$ EDTA, 10\% glycerol) for 20 minutes on ice. After centrifugation at $4^{\circ} \mathrm{C}$ at 20,000 $\mathrm{g}$, the supernatant was collected as the nuclear extract (57).

Immunoblot analysis. The primary antibodies used were: ATF6 (ab11909, Abcam); Rieske (ab14746, Abcam); GGDPS (MA5-25243, Abcam); p-STAT6, clone 19E2.1 (MABE270, MilliporeSigma), Rac1, clone 23A8 (05-389, MilliporeSigma), surfactant protein C (SPC) (AB3786, MilliporeSigma); BiP (3183, Cell Signaling Technology [CST]), HIF-2 $\alpha$ (7096, CST), KLF4 (4038, CST), lamin A/C (2032, CST), PPAR $\gamma$ (2430, CST), and voltage-dependent anion channel (VDAC) (4866, CST); GGTase I (sc-376655, Santa Cruz Biotechnology); $\beta$-actin (A5441, MilliporeSigma), Flag (F1804, MilliporeSigma); and $\alpha$-SMA (03-61001, American Research Products).

ELISA. TGF- $\beta 1$, TNF- $\alpha$, IL-10, MCP-1, YM-1, and PDGF-BB expression were determined in BALF using ELISA kits (R\&D Systems) according to the manufacturer's instructions.

GTPase activity. Rac1, Rac2, and RhoA activity was determined with the G-LISA Kit (Cytoskeleton) according to the manufacture's protocol using the provided antibody or an anti-Rac2 antibody (07604-I) from MilliporeSigma.

Arginase 1 activity. Arginase 1 activity was determined using the QuantiChrom Arginase Assay Kit (BioAssay Systems) according to the manufacturer's instructions.

Caspase-3 activity. Caspase-3 activity was measured using the EnzChek Caspase-3 Assay Kit Number 2 (Molecular Probes) according to the manufacturer's protocol. Cells were lysed in $1 \times$ lysis buffer, subjected to a freeze-thaw cycle, centrifuged to remove cellular debris, and loaded into individual microplate wells. The $2 \times$ reaction buffer with substrate was immediately added to the samples, and fluorescence was measured (excitation/emission, $496 \mathrm{~nm} / 520 \mathrm{~nm}$ ). A supplied inhibitor was used as a negative control in all experiments (31).

Total cholesterol assay. Total cholesterol was determined using a colorimetric Total Cholesterol Assay Kit (Cell Biolabs Inc.) according to the manufacturer's protocol.

Triton $X$-114 separation. Rac1 isoprenylation was determined by lysing cells in ice-cold Triton X-114 lysis buffer (20 mM Tris, pH 7.5, $150 \mathrm{mM} \mathrm{NaCl}$, and 1\% Triton X-114). Cell lysates were sonicated and cleared by centrifugation. The supernatant was incubated at $37^{\circ} \mathrm{C}$ for 10 minutes and then centrifuged at room temperature for 2 minutes at $12,000 \mathrm{~g}$. The detergent phase (prenylated) was diluted with buffer 
that did not contain Triton X-114, and the aqueous phase (unprenylated) was transferred to a new tube (31).

TGF- $\beta 1$ neutralization. Conditioned media from macrophages treated with vehicle or GGOH $(50 \mu \mathrm{M})$ overnight were incubated with IgG or TGF- $\beta 1-$ neutralizing antibody $(10 \mu \mathrm{g} / \mathrm{mL})$. Fibroblasts were harvested after 24 hours of incubation with conditioned media (9).

Hydroxyproline analysis. Lung, liver, and kidney tissues were dried to a stable weight and acid hydrolyzed with $6 \mathrm{~N} \mathrm{HCl}$ for 24 hours at $110^{\circ} \mathrm{C}$. Samples were resuspended in $1.5 \mathrm{~mL}$ PBS followed by incubation at $60^{\circ} \mathrm{C}$ for 1 hour. Samples were centrifuged at $18,000 \mathrm{~g}$, and the supernatant was taken for hydroxyproline analysis using chloramine-T. The hydroxyproline concentration was normalized to the dry weight of the tissue.

IHC. Mouse lung sections (4- $\mu$ m-thick) were prepared, deparaffinized with xylene, and rehydrated with a graded series of ethanol (absolute; 95\%, 90\%, 80\%, and 70\% in water). The slides were incubated in ice-cold permeabilization solution (0.1\% Triton X-100 and $0.1 \%$ sodium citrate in water) and blocked in PBS containing $10 \%$ BSA and $10 \%$ normal goat serum. Sections were stained with rabbit antiSPC polyclonal antibody (AB3786, MilliporeSigma) and goat anti-rabbit IgG-FITC (4030-02, Southern Biotech), followed by TUNEL staining (12156792910, MilliporeSigma) and then counterstaining with DAPI. Images were captured with a Nikon A1 confocal microscope.

Statistics. Statistical comparisons were performed using a Student's $t$ test when only 2 groups of data were analyzed or 1-way ANOVA with Tukey's post hoc test or 2-way ANOVA followed by Bonferroni's post hoc test when multiple data groups were analyzed. Correlation analysis was performed using Pearson's correlation. All statistical analyses are expressed as the mean \pm SEM, and a $P$ value of less than 0.05 was considered statistically significant. GraphPad Prism 5.0 (GraphPad Software) statistical software was used for all analyses.

Study approval. BAL cells were obtained from healthy subjects and from subjects with IPF under protocol no. 300001124 approved by the Human Subjects Institutional Review Board of the University of Alabama at Birmingham (UAB). Human BAL specimens were used for research only. All subjects provided written consent prior to their participation in the study. Animal experiments were approved by the IACUC of UAB under protocol nos. 21076 and 21149 and performed in accordance with NIH guidelines.

\section{Author contributions}

JLLC and ABC developed the concept and design of the study. JLLC, LG, DD, MV, VSH, CH, JDN, RJH, GQC, QD, RS, VBA, ALFL, KAZ, TFB, LSW, SB, and ABC helped conduct the experiments. JLLC, LG, DD, MV, VSH, ALFL, KAZ, GQC, RS, TFB, LSW, JDN, RJH, and ABC acquired data. JLLC, JDN, RJH, QD, VBA, SB, $\mathrm{BKY}$, and $\mathrm{ABC}$ provided reagents. JLLC, LG, MV, VSH, JDN, RJH, QD, RS, VBA, ALFL, KAZ, BKY, TFB, LSW, SB, and ABC provided analysis and interpretation of experiments and results. JLLC and $\mathrm{ABC}$ wrote the manuscript.

\section{Acknowledgments}

Chrysotile asbestos was a gift from Peter S. Thorne (University of Iowa, Iowa City, Iowa). Research reported in this publication was supported by NIH grants (2R01ES015981-12, 1R56ESO27464-01, and 1 I01 CX001715-01, to ABC) from the Department of Veteran Affairs and a Parker B. Francis fellowship and American Lung Association grant (RG-507440, to JLC). Support for the Comprehensive Flow Cytometry Core was provided by NIH grants (P30AR048311 and P3OAI27667). The AB SCIEX 6500 mass spectrometer in the Targeted Metabolomics and Proteomics Laboratory was purchased with funds provided by the UAB Health Services Foundation General Endowment Fund and operational funds from the UAB O'Brien Acute Kidney Injury Center (P30DK079337) and the UAB Lung Health Center.

Address correspondence to: A. Brent Carter, 1918 University Boulevard, 404 MCLM; Pulmonary, Allergy, and Critical Care Medicine; University of Alabama at Birmingham, Birmingham, Alabama 35294, USA. Phone: 205.996.1682; Email: bcarter1@uab.edu.
1. Hutchinson JP, McKeever TM, Fogarty AW, Navaratnam V, Hubbard RB. Increasing global mortality from idiopathic pulmonary fibrosis in the twenty-first century. Ann Am Thorac Soc. 2014;11(8):1176-1185.

2. King TE, et al. A phase 3 trial of pirfenidone in patients with idiopathic pulmonary fibrosis. N Engl J Med. 2014;370(22):2083-2092.

3. Richeldi L, et al. Efficacy and safety of nintedanib in idiopathic pulmonary fibrosis. $\mathrm{NEngl} \mathrm{J} \mathrm{Med.}$ 2014;370(22):2071-2082.

4. Crystal RG, Bitterman PB, Rennard SI, Hance AJ, Keogh BA. Interstitial lung diseases of unknown cause. Disorders characterized by chronic inflammation of the lower respiratory tract (first of two parts). N Engl JMed.1984;310(3):154-166.

5. Korfei M, et al. Epithelial endoplasmic reticulum stress and apoptosis in sporadic idiopathic pulmonary fibrosis. Am J Respir Crit Care Med. 2008;178(8):838-846.

6. Kim KK, et al. Alveolar epithelial cell mesenchymal transition develops in vivo during pulmonary fibrosis and is regulated by the extracellular matrix. Proc Natl Acad Sci U S A.
2006;103(35):13180-13185.

7. Gross TJ, Hunninghake GW. Idiopathic pulmonary fibrosis. N Engl J Med. 2001;345(7):517-525.

8. He C, Ryan AJ, Murthy S, Carter AB. Accelerated development of pulmonary fibrosis via $\mathrm{Cu}, \mathrm{Zn}$-superoxide dismutase-induced alternative activation of macrophages. J Biol Chem. 2013;288(28):20745-20757.

9. Larson-Casey JL, Deshane JS, Ryan AJ, Thannickal VJ, Carter AB. Macrophage Akt1 kinase-mediated mitophagy modulates apoptosis resistance and pulmonary fibrosis. Immunity. 2016;44(3):582-596.

10. Duffield JS, et al. Selective depletion of macrophages reveals distinct, opposing roles during liver injury and repair. JClin Invest. 2005;115(1):56-65.

11. Redente EF, et al. Tumor necrosis factor- $\alpha$ accelerates the resolution of established pulmonary fibrosis in mice by targeting profibrotic lung macrophages. Am J Respir Cell Mol Biol. 2014;50(4):825-837.

12. He C, Larson-Casey JL, Gu L, Ryan AJ, Murthy S, Carter AB. Cu,Zn-superoxide dismutasemediated redox regulation of jumonji domain containing 3 modulates macrophage polarization and pulmonary fibrosis. Am J Respir Cell Mol Biol. 2016;55(1):58-71.

13. Gu L, Larson-Casey JL, Carter AB. Macrophages utilize the mitochondrial calcium uniporter for profibrotic polarization. FASEB J. 2017;31(7):3072-3083.

14. Murthy S, Larson-Casey JL, Ryan AJ, He C, Kobzik L, Carter AB. Alternative activation of macrophages and pulmonary fibrosis are modulated by scavenger receptor, macrophage receptor with collagenous structure. FASEB J. 2015;29(8):3527-3536.

15. Hosfield DJ, et al. Structural basis for bisphosphonate-mediated inhibition of isoprenoid biosynthesis. JBiol Chem. 2004;279(10):8526-8529.

16. Dan HC, et al. Phosphatidylinositol-3-OH kinase/AKT and survivin pathways as critical targets for geranylgeranyltransferase I inhibitorinduced apoptosis. Oncogene. 2004;23(3):706-715.

17. Sjogren AK, et al. GGTase-I deficiency reduces tumor formation and improves survival in mice with K-RAS-induced lung cancer. JClin Invest. 2007;117(5):1294-1304.

18. Amano M, et al. Identification of a putative target 
for Rho as the serine-threonine kinase protein kinase N. Science. 1996;271(5249):648-650.

19. Kaminski N, et al. Global analysis of gene expression in pulmonary fibrosis reveals distinct programs regulating lung inflammation and fibrosis. Proc Natl Acad Sci USA. 2000;97(4):1778-1783.

20. Bopp A, Wartlick F, Henninger C, Kaina B, Fritz G. Rac1 modulates acute and subacute genotoxininduced hepatic stress responses, fibrosis and liver aging. Cell Death Dis. 2013;4:e558.

21. Lavall D, Schuster P, Jacobs N, Kazakov A, Böhm M, Laufs U. Rac1 GTPase regulates $11 \beta$ hydroxysteroid dehydrogenase type 2 and fibrotic remodeling. J Biol Chem. 2017;292(18):7542-7553.

22. Osborn-Heaford HL, et al. Mitochondrial Rac1 GTPase import and electron transfer from cytochrome $\mathrm{c}$ are required for pulmonary fibrosis. J Biol Chem. 2012;287(5):3301-3312.

23. Lawson WE, et al. Endoplasmic reticulum stress enhances fibrotic remodeling in the lungs. Proc Natl Acad Sci U S A. 2011;108(26):10562-10567.

24. Misharin AV, et al. Monocyte-derived alveolar macrophages drive lung fibrosis and persist in the lung over the life span. J Exp Med. 2017;214(8):2387-2404.

25. Murthy S, Ryan A, He C, Mallampalli RK, Carter AB. Rac1-mediated mitochondrial $\mathrm{H} 2 \mathrm{O} 2$ generation regulates MMP-9 gene expression in macrophages via inhibition of SP-1 and AP-1. J Biol Chem. 2010;285(32):25062-25073.

26. Heller NM, et al. Type I IL-4Rs selectively activate IRS-2 to induce target gene expression in macrophages. Sci Signal. 2008;1(51):ra17.

27. Takeda N, et al. Differential activation and antagonistic function of HIF-\{alpha\} isoforms in macrophages are essential for NO homeostasis. Genes Dev. 2010;24(5):491-501.

28. Szanto A, et al. STAT6 transcription factor is a facilitator of the nuclear receptor PPAR $\gamma$-regulated gene expression in macrophages and dendritic cells. Immunity. 2010;33(5):699-712.

29. Liao X, et al. Krüppel-like factor 4 regulates macrophage polarization. J Clin Invest. 2011;121(7):2736-2749.

30. Odegaard JI, et al. Macrophage-specific PPARgamma controls alternative activation and improves insulin resistance. Nature. 2007;447(7148):1116-1120.

31. Larson-Casey JL, Murthy S, Ryan AJ, Carter AB. Modulation of the mevalonate pathway by akt regulates macrophage survival and development of pulmonary fibrosis. J Biol Chem.
2014;289(52):36204-36219.

32. Xia H, et al. Pathological integrin signaling enhances proliferation of primary lung fibroblasts from patients with idiopathic pulmonary fibrosis. J Exp Med. 2008;205(7):1659-1672.

33. Surolia R, et al. 3D pulmospheres serve as a personalized and predictive multicellular model for assessment of antifibrotic drugs. JCI Insight. 2017;2(2):e91377.

34. Ge J, et al. RhoA, Rac1, and Cdc42 differentially regulate $\alpha \mathrm{SMA}$ and collagen I expression in mesenchymal stem cells. J Biol Chem. 2018;293(24):9358-9369.

35. Carano A, Teitelbaum SL, Konsek JD, Schlesinger $\mathrm{PH}$, Blair HC. Bisphosphonates directly inhibit the bone resorption activity of isolated avian osteoclasts in vitro. J Clin Invest. 1990;85(2):456-461.

36. Kazi A, et al. Blockade of protein geranylgeranylation inhibits Cdk2-dependent p27Kip1 phosphorylation on Thr187 and accumulates p27Kip1 in the nucleus: implications for breast cancer therapy. Mol Cell Biol. 2009;29(8):2254-2263.

37. Sun J, et al. Geranylgeranyltransferase I inhibitor GGTI-2154 induces breast carcinoma apoptosis and tumor regression in $\mathrm{H}$-Ras transgenic mice. Cancer Res. 2003;63(24):8922-8929.

38. Alexeeff SE, Litonjua AA, Sparrow D, Vokonas PS, Schwartz J. Statin use reduces decline in lung function: VA Normative Aging Study. Am J Respir Crit Care Med. 2007;176(8):742-747.

39. Kreuter M, et al. Effect of statins on diseaserelated outcomes in patients with idiopathic pulmonary fibrosis. Thorax. 2017;72(2):148-153.

40. Kreuter M, et al. Statin therapy and outcomes in trials of nintedanib in idiopathic pulmonary fibrosis. Respiration. 2018;95(5):317-326.

41. Saad N, Camus P, Suissa S, Ernst P. Statins and the risk of interstitial lung disease: a cohort study. Thorax. 2013;68(4):361-364.

42. Nadrous HF, Ryu JH, Douglas WW, Decker PA, Olson EJ. Impact of angiotensin-converting enzyme inhibitors and statins on survival in idiopathic pulmonary fibrosis. Chest. 2004;126(2):438-446.

43. Fernández AB, Karas RH, Alsheikh-Ali AA, Thompson PD. Statins and interstitial lung disease: a systematic review of the literature and of food and drug administration adverse event reports. Chest. 2008;134(4):824-830.

44. Xu JF, et al. Statins and pulmonary fibrosis: the potential role of NLRP3 inflammasome activation. Am J Respir Crit Care Med. 2012;185(5):547-556.
45. Chen X, et al. Integrin alpha1beta1 controls reactive oxygen species synthesis by negatively regulating epidermal growth factor receptor-mediated Rac activation. Mol Cell Biol. 2007;27(9):3313-3326.

46. Didsbury J, Weber RF, Bokoch GM, Evans T, Snyderman R. rac, a novel ras-related family of proteins that are botulinum toxin substrates. J Biol Chem. 1989;264(28):16378-16382.

47. Kheradmand F, Werner E, Tremble P, Symons $\mathrm{M}$, Werb Z. Role of Rac1 and oxygen radicals in collagenase-1 expression induced by cell shape change. Science. 1998;280(5365):898-902.

48. Zeng PY, Rane N, Du W, Chintapalli J, Prendergast GC. Role for RhoB and PRK in the suppression of epithelial cell transformation by farnesyltransferase inhibitors. Oncogene. 2003;22(8):1124-1134.

49. Kalluri R, Neilson EG. Epithelial-mesenchymal transition and its implications for fibrosis. J Clin Invest. 2003;112(12):1776-1784.

50. Lauriol J, et al. RhoA signaling in cardiomyocytes protects against stress-induced heart failure but facilitates cardiac fibrosis. Sci Signal. 2014;7(348):ra100.

51. Sawada N, et al. Cyclic GMP kinase and RhoA Ser188 phosphorylation integrate pro- and antifibrotic signals in blood vessels. Mol Cell Biol. 2009;29(22):6018-6032.

52. Zhou Y, et al. Inhibition of mechanosensitive signaling in myofibroblasts ameliorates experimental pulmonary fibrosis. J Clin Invest. 2013;123(3):1096-1108.

53. Pittet JF, et al. TGF-beta is a critical mediator of acute lung injury. J Clin Invest. 2001;107(12):1537-1544.

54. Glogauer M, et al. Rac1 deletion in mouse neutrophils has selective effects on neutrophil functions. JImmunol. 2003;170(11):5652-5657.

55. Erickson JR, et al. A dynamic pathway for calciumindependent activation of CaMKII by methionine oxidation. Cell. 2008;133(3):462-474.

56. Ridge KM, Rutschman DH, Factor P, Katz AI, Bertorello AM, Sznajder JL. Differential expression of Na-K-ATPase isoforms in rat alveolar epithelial cells. Am J Physiol. 1997; 273(1 Pt 1):L246-L255.

57. Carter AB, Hunninghake GW. A constitutive active MEK --> ERK pathway negatively regulates NF-kappa B-dependent gene expression by modulating TATA-binding protein phosphorylation. J Biol Chem. 2000;275(36):27858-27864. 